KEK-TH-962

\title{
One-loop anomalous couplings and matching conditions in the nonlinear realization of the $\mathrm{SU}(2)$ Higgs Model \\ Sukanta Dutta*, Kaoru Hagiwara and Qi-Shu Yan ${ }^{\dagger}$ \\ Theory Group, KEK, Tsukuba, 305-0801, Japan
}

We study the renormalization of the nonlinear realization of the $\mathrm{SU}(2)$ Higgs model in the modified minimal subtraction renormalization scheme. We propose that the effective field method with truncated operator series is trustworthy even when the Higgs boson is relatively light. Using the technique of background field method in the coordinate space, we derive the matching conditions at tree and one loop order, both in the regions where the Higgs boson is heavy and where it is light. We obtain the complete one-loop anomalous couplings up to $O\left(p^{4}\right)$ in the SU(2) Higgs model. We observe that the contribution of the gauge bosons and the Goldstone bosons to the anomalous couplings at the one-loop level is significant. By establishing the correspondence between our coordinate space calculation and the momentum space calculation that exists in the literature, we find agreement in all the matching conditions in the heavy Higgs boson limit.

PACS: 11.10.Gh, 11.10.Hi

\section{INTRODUCTION}

In this paper, we study the renormalization of the $S U(2)$ Higgs model in the non-linear realization, and demonstrate how the matching condition up to the one-loop level in the $S U(2)$ chiral Lagrangian is made, as prescribed in [1]. Such a toy model has been studied in the reference [2] to investigate the decoupling limit of a heavy Higgs boson using the path integral method.

\footnotetext{
*Email Address: Sukanta.Dutta@kek.jp

(On leave from SGTB Khalsa College, University of Delhi. Delhi-110007. India )

†Email Address: yanqs@post.kek.jp
} 
The chiral Lagrangian method is one of the important derivatives of the effective field theory method, and has been used for the description of both the hadronic [3] and electroweak physics [4]. Considering the fact that the chiral Lagrangian for electroweak physics contains more than ten extra operators, before examining the chiral electroweak $S U(2) \times U(1)$ model, here we will study the simplest and non-trivial toy model: the $S U(2)$ Higgs model and its chiral effective Lagrangian with spontaneous symmetry breaking.

We will use the modified minimal subtraction scheme $(\overline{M S})$ in our calculation. It is a more convenient renormalization scheme, since the divergences can be automatically subtracted and expressed in a simple form. With the right matching procedure in the $\overline{M S}$, the infrared divergences of the effective theory are just the same as those of the full theory. And that's why it is regarded as one of the pillars of the effective field theory method $[1,5]$.

According to the procedure specified for the effective field theory [1], the matching conditions are obtained order by order at the matching scale. To the n-loop order they can be expressed as

$$
\int \delta \mathcal{L}^{n}(\phi)=S_{\text {Full }}^{n}(\phi, \Phi[\phi])-S_{\text {eff }}^{n}(\phi),
$$

where $\phi$ corresponds to the degrees of freedom at low energy region, and $\Phi$ corresponds to the heavy degrees of freedom, which at the matching scale is expressed in terms of $\phi$ through its equation of motion (EOM). The terms $S_{F u l l}^{n}(\phi, \Phi[\phi])$ and $S_{\text {eff }}^{n}(\phi)$ are the effective actions of the full and the effective theories, respectively, up to $n$-loop order. The term $\delta \mathcal{L}^{n}(\phi)$ accounts for the n-loop matching conditions, which determines the anomalous couplings at $n$-loop order.

It is known in the literature, that there are two different (but intrinsically the same) methods to construct the low energy effective Lagrangian from a full renormalizable theory. The first method is the standard diagrammatic one, i.e. to compute the one particle irreducible Feynman diagrams, and then to match them order by order with those computed in the effective theory. The authors of [6] have used this method to extract the non-decoupling terms for a heavy Higgs boson in the $S U(2) \times U(1)$ case. And the authors of [7] used it to consider one of the extensions of the standard model with a heavy scalar singlet coupled to the leptonic doublet.

The second method is the path integral, in which the effects of the heavy degrees of freedom can also be extracted order by order. For example in the one-loop case, the heavy degrees of freedom can be integrated out formally by the Gaussian integration and we can compute the renormalization, purely with the algebraic manipulation and without any use of the technique of Feynman diagrams. However, the second method is equivalent to the 
first one, since it only combines the Feynman diagrams in a specific way (to guarantee the gauge invariance in the gauge theories). We can still utilize the Feynman diagram method to guide our calculations. The authors of $[2,8]$ have explored this method by deriving the non-decoupling effects in the decoupling limit of a heavy Higgs boson in both the $S U(2)$ and $S U(2) \times U(1)$ cases. By using this method, in the reference [9], the renormalization group equations (RGE) of the effective chiral SU(2) Lagrangian are obtained, which will be used for comparison in this article.

We would like to make some comments on these works:

1. All previous studies on the chiral Lagrangian theories for the bosonic sector derived the non-decoupling effect of the Higgs boson, in the decoupling limit. In this limit one can evade the evaluation prescription for the matching conditions given by the Eq. (1), by ignoring the term $\mathcal{L}_{\text {eff }}^{1-\text { loop }}$ which is suppressed by Higgs boson's mass. However these studies are not applicable to the light Higgs boson scenarios. The authors of reference [9] found that when Higgs boson is relatively light, the predictions of the RGE method deviate from the prediction of the decoupling limit significantly.

2. Although the references $[2,8]$ worked in the non-linear realization, they have not extracted all divergences of the one loop effective Lagrangian. Therefore, the demonstration of the renormalizability of these models in the non-linear realization is still missing.

3. The parameterization in the reference [2] generates D'Alambert operator for the Goldstone bosons given as

$$
\square^{a b}=\frac{(v+\bar{H})^{2}}{v^{2}}\left\{\left[-(d \cdot d)^{a b}+m_{W}^{2} \delta^{a b}\right]+\sigma_{\xi}^{a b}\right\},
$$

which leads to an ill-defined propagator (where $m_{W}$ is the gauge boson mass and the definitions for $(d \cdot d)^{a b}$ and $\sigma_{\xi}^{a b}$ are given later in section 4 and Eq. (4.11b), respectively. This ill-defined propagator introduces the quartic and the quadratic divergences and makes it difficult to verify the renormalizability of these models.

Therefore, it is worthwhile to study the renormalization of the non-linear $S U(2)$ Higgs model in the $\overline{M S}$ renormalization scheme, and to derive the matching conditions up to one-loop level which is valid for a large range of Higgs boson's mass. We also examine the renormalization scale dependence of the one-loop anomalous couplings. 
Just for the sake of convenience, we conduct all our calculation in the Euclidean coordinate space-time. As the matter of fact, not only for the divergent terms as mentioned in [10], but also for the finite terms, it is less cumbersome to work in the coordinate space-time.

This paper is organized as follows. Section 2, briefly introduces the Lagrangian $\mathcal{L}_{S U(2)}$ of the renormalizable $S U(2)$ Higgs model (full theory), and concentrate on its nonlinear form. Section 3, introduces the nonlinear effective $S U(2)$ Lagrangian $L^{\text {eff }}$ up to $O\left(p^{4}\right)$. In section 4 , we perform the renormalization of the $\mathcal{L}_{S U(2)}$ in the background field method (BFM). In section 5, the complete one-loop anomalous couplings in the SU(2) Higgs model are calculated. Section 6 presents the tree level and the one-loop level matching conditions. In section 7 , we list the the one-loop RGE calculation in the effective Lagrangian. In section 8, numerical analysis is given to compare the effective couplings obtained by RGE method, those calculated in the decoupling limit [2] and those following from the exact oneloop calculation of the renormalizable SU(2) Higgs model. We summarize this article with discussions and conclusions. We provide the basics of the short distance approximation in the appendix $\mathrm{A}$. In appendix $\mathrm{B}$ and $\mathrm{C}$ we provide the necessary mathematical tool to extract the divergent and the finite parts from the effective generating functionals of SU(2) Higgs model and the effective SU(2) chiral Lagrangian respectively. Appendix D addresses the definition of the scalar loop integral in coordinate space formalism.

\section{THE RENORMALIZABLE $S U(2)$ HIGGS MODEL}

The partition functional of the renormalizable non-Abelian $S U(2)$ Higgs model [11] (This does not include the gauge fixing and ghost terms. They will be added when necessary for the quantization of quantum fields ) can be expressed as

$$
\mathcal{Z}=\int \mathcal{D} A_{\mu}^{a} \mathcal{D} \phi \mathcal{D} \phi^{\dagger} \exp \left(\mathcal{S}\left[A, \phi, \phi^{\dagger}\right]\right),
$$

where the action $\mathcal{S}$ is determined by the following Lagrangian density

$$
\mathcal{L}=-\frac{1}{4 g^{2}} W_{\mu \nu}^{a} W^{a \mu \nu}-(D \phi)^{\dagger} \cdot(D \phi)+\mu^{2} \phi^{\dagger} \phi-\frac{\lambda}{4}\left(\phi^{\dagger} \phi\right)^{2},
$$

and the definition of quantities in this Lagrangian is given below

$$
\begin{aligned}
W_{\mu \nu}^{a} & =\partial_{\mu} W_{\nu}^{a}-\partial_{\nu} W_{\mu}^{a}+f^{a b c} W_{\mu}^{b} W_{\nu}^{c}, \\
D_{\mu} \phi & =\partial_{\mu} \phi-i W_{\mu}^{a} T^{a} \phi, \\
\phi^{\dagger} & =\left(\phi_{1}^{*}, \phi_{2}^{*}\right),
\end{aligned}
$$


where $T^{a}$ is the generator of the Lie algebra of $S U(2)$ gauge group.

The spontaneous symmetry breaking is induced by the positive mass square $\mu^{2}$ in the Higgs potential. The vacuum expectation value of Higgs field is given as $|\langle\phi\rangle|=v / \sqrt{2}$. And by eating the corresponding Goldstone boson, the gauge bosons $W$ obtain their masses.

The non-linear realization of the Lagrangian given in Eq. (2.2) is made by changing the variable $\phi$

$$
\phi=\frac{1}{\sqrt{2}}(v+H) U, U=\exp \left(i \zeta^{a} T^{a}\right), v=2 \sqrt{\frac{\mu^{2}}{\lambda}},
$$

where the matrix field $U$ is the Goldstone boson field as prescribed by the Goldstone theorem, $\zeta^{a}$ is the dimensionless phase angle, and the $H$ is a massive scalar field. Then the Lagrangian in Eq. (2.2) is rewritten as

$$
\begin{aligned}
\mathcal{L}^{\prime}= & -\frac{1}{4 g^{2}} W_{\mu \nu}^{a} W^{a \mu \nu}-\frac{(v+H)^{2}}{4} \operatorname{tr}\left[(D U)^{\dagger} \cdot(D U)\right] \\
& -\frac{1}{2} \partial H \cdot \partial H+\frac{1}{2} \mu^{2}(v+H)^{2}-\frac{\lambda}{16}(v+H)^{4},
\end{aligned}
$$

where $t r$ is sum over the $\mathrm{SU}(2)$ group indices. And the change of variables induces a determinant factor in the functional integral $\mathcal{Z}$

$$
\mathcal{Z}=\int \mathcal{D} W_{\mu}^{a} \mathcal{D} H \mathcal{D} \zeta^{b} \exp \left(\mathcal{S}^{\prime}[W, H, \xi]\right) \operatorname{det}\{(v+H) \delta(x-y)\}
$$

The determinant can be written in the exponential form, and correspondingly the Lagrangian density is modified to

$$
\mathcal{L} \rightarrow \mathcal{L}^{\prime}+\delta(0) \ln \{v+H\}
$$

There exists an arbitrariness to express the phase angle $\zeta^{a}$ into a field. However, this arbitarariness should not change the physics [12]. According to our experience in the hadronic chiral Lagrangian, the relation between the phase angle $\zeta^{a}$ and the Goldstone field $\xi^{a}$ can be defined as

$$
\zeta^{a}=\frac{2 \xi^{a}}{v}
$$

Then the partition function $Z$ can be expressed by quantum fields as

$$
\mathcal{Z}=\int \mathcal{D} W_{\mu}^{a} \mathcal{D} H \mathcal{D} \xi^{b} \exp \left(\mathcal{S}^{\prime}[W, H, \xi]\right) \operatorname{det}\left\{\left(1+\frac{H}{v}\right) \delta(x-y)\right\} .
$$

Now the Lagrangian density is modified to the following form 


$$
\mathcal{L} \rightarrow \mathcal{L}^{\prime}+\delta(0) \ln \left\{1+\frac{H}{v}\right\}
$$

This determinant which contains quartic divergences is indispensable and crucial to cancel exactly the quartic divergences brought into by the longitudinal part of gauge boson, and is important in verifying the renormalizability of the Higgs model in the U-gauge $[13,14]$.

\section{THE NON-RENORMALIZABLE EFFECTIVE CHIRAL SU(2) THEORY UP TO $O\left(P^{4}\right)$}

Any effective Lagrangian is valid within its infrared cutoff $\Lambda_{I R}$ and its ultraviolet cutoff $\Lambda_{U V}$. In the nonlinear chiral effective $S U(2)$ Lagrangian $L^{\text {eff }}$, the effective dynamic degrees of freedom at low energy region includes only the Goldstone and the gauge bosons. Masses of the quantum Goldstone bosons are gauge dependent, and can be vanishing or infinitely heavy. But the masses of the gauge bosons are fixed by the experiments. So the infrared cutoff $\Lambda_{I R}$ for this effective Lagrangian should be larger than the masses of the gauge bosons at the low energy region in the theory. However, this fact invalidates the naive momentum power counting rule in the hadronic chiral Lagrangian, where only massless Goldstone bosons are included in the theory and the momentum $p$ is assumed to be much smaller than the vacuum expectation value $v$. For the ultraviolet cutoff $\Lambda_{U V}$ we know that it should be lower than the new resonances otherwise the new degrees of freedom would break the validity of the effective theory.

In principle the Lagrangian $L^{e f f}$, which includes all permitted operators composed by these light degrees of freedom and respects the assumed Lorentz and gauge symmetries, is still renormalizable [15]. But for the realistic renormalization procedure the following facts serves as the important guidelines. 1) The Wilsonian renormalization method [16] and the surface theorem [17] which reveal that, only few operators play important role to determine the behavior of the dynamic system at the low energy region, enable us to truncate the infinite divergence tower up to a specified order and to consider the renormalization of the effective Lagrangian order by order; 2) While in the dimensional regularization method, although the quartic divergences exist in the loop calculation, it allows us to express them to be proportional to the masses in the theory, as done with the quadratic divergences.

The effective couplings of operators in the Lagrangian $L^{\text {eff }}$ form a parameter space of the effective Lagrangian, and they effectively reflect the dynamics of the underlying theories and the behavior of symmetry breaking. Different underlying theories and ways of symmetry breaking will fall into this effective parameter space as special points. When 
the scale runs from the high energy region down to the low energy region, a characteristic curve in this parameter space is obtained. If we can measure or extract this curve from the interpretation and extrapolation of experimental results, then it would help us to figure out the possible underlying theories, as we attempt to do in speculating the grand unification theories.

The magnitudes of these effective couplings are arbitrary. But generally speaking, the unitarity condition for the theoretical prediction puts a constraint on the magnitudes of the effective couplings of these operators. And the larger the ultraviolet cutoff $\Lambda_{U V}$ of the theory is, the smaller are the magnitudes of the effective couplings of these operators [9]. However, here in order to keep the generality and universality of the effective Lagrangian method, we do not make any assumption on the magnitude of these effective couplings and simply classify operators with the same mass dimensions as the same order.

For the $S U(2)$ case, the general effective $S U(2)$ Lagrangian $L^{\text {eff }}$ which is consistent with the Lorentz spacetime symmetry, $S U(2)$ gauge symmetry, and the charge, parity, and the combined CP symmetries, can be formulated as

$$
\begin{aligned}
\mathcal{L}_{\text {eff }}= & \mathcal{L}_{2}+\mathcal{L}_{4}+\mathcal{L}_{6}+\cdots, \\
\mathcal{L}_{2}= & \frac{v^{2}}{4} \operatorname{tr}\left[V_{\mu} V^{\mu}\right], \\
\mathcal{L}_{4}= & -\frac{1}{4 g^{2}} W_{\mu \nu}^{a} W^{a \mu \nu}-i d_{1} \operatorname{tr}\left[W_{\mu \nu} V^{\mu} V^{\nu}\right] \\
& +d_{2} \operatorname{tr}\left[V_{\mu} V_{\nu}\right] \operatorname{tr}\left[V^{\mu} V^{\nu}\right]+d_{3} \operatorname{tr}\left[V_{\mu} V^{\mu}\right] \operatorname{tr}\left[V_{\nu} V^{\nu}\right], \\
\mathcal{L}_{6}= & \cdots,
\end{aligned}
$$

where the $\mathcal{L}_{2}$ and $\mathcal{L}_{4}$ represent the relevant and marginal operators in the Wilsonian renormalization method, respectively. Henceforth for the simplicity, we omit all the irrelevant operators in our consideration, i.e. higher dimensional operators greater than $O\left(p^{4}\right)$. The operators in the $\mathcal{L}_{2}$ and $\mathcal{L}_{4}$ also form the set of complete operators up to $O\left(p^{4}\right)$ in the usual momentum counting rule. And the higher dimension ( irrelevant ) operators greater than $O\left(p^{4}\right)$ order are contained in $\mathcal{L}_{6}$. The auxiliary variable $V_{\mu}$ is defined as

$$
V_{\mu}=U^{\dagger} D_{\mu} U, D_{\mu} U=\partial_{\mu} U-i W_{\mu} U
$$

to simplify the representation. Due to the following relations of the $S U(2)$ gauge group

$$
\operatorname{tr}\left[T^{a} T^{b} T^{c} T^{d}\right]=\frac{1}{8}\left(\delta^{a b} \delta^{c d}+\delta^{a d} \delta^{b c}-\delta^{a c} \delta^{b d}\right),
$$

the terms, like $\operatorname{tr}\left[V_{\mu} V_{\nu} V^{\mu} V^{\nu}\right]$ and $\operatorname{tr}\left[V_{\mu} V^{\mu} V_{\nu} V^{\nu}\right]$, can be linearly composed by $\operatorname{tr}\left[V_{\mu} V_{\nu}\right] \operatorname{tr}\left[V^{\mu} V^{\nu}\right]$ and $\operatorname{tr}\left[V_{\mu} V^{\mu}\right] \operatorname{tr}\left[V_{\nu} V^{\nu}\right]$. And since here we do not consider the operators which break the charge, or parity, or both symmetries, therefore, the operators in 
Eq. (3.1c) are complete and linearly independent. Before havng any certain idea on the magnitude of $d_{i}$, which does not possess any mass dimension, we can classify them in the same class of the effective couplings as that of $1 / g^{2}$.

\section{THE RENORMALIZATION OF THE NONLINEAR SU(2) HIGGS MODEL BY BFM}

We formulate the partition function of the nonlinear $S U(2)$ Higgs model in Eq.(2.12) in the BFM as

$$
\begin{aligned}
\mathcal{Z}\left[\bar{W}^{s}, \bar{H}\right]= & \int \mathcal{D} \widehat{W}_{\mu}^{a} \mathcal{D} \widehat{H} \mathcal{D} \widetilde{\xi}^{b} \operatorname{det}\left\{\left(\frac{v+\bar{H}+\widehat{H}}{v}\right) \delta(x-y)\right\} \\
& \exp \left(\mathcal{S}^{\prime}\left[\bar{W}^{s}, \bar{H} ; \widehat{W}, \widehat{H}, \widetilde{\xi}\right]+S_{G F}^{\prime}\right),
\end{aligned}
$$

where the classical field $\bar{W}^{s}$ is given as

$$
\bar{W}^{s}=\bar{U}^{\dagger} \overline{W U}+i \bar{U}^{\dagger} \partial \bar{U} .
$$

From now on, in order to simplify expressions, we will abuse $\bar{W}$ to represent $\bar{W}^{s}$. And $\bar{U}$ and $\bar{H}$ are the classical parts of the Goldstone and Higgs fields, respectively. The quantum fields $\widehat{W}_{\mu}^{a}, \widehat{H}$, and $\widetilde{\xi}^{b}$ are the quantum parts of gauge, scalar, and Goldstone fields. In the BFM [18], we can choose different gauges for the classical and quantum fields, respectively. Although the classical fields might be restricted by their classical EOM, the gauge fixing condition is still needed in order to find one unique solution, as we do in the classical electrodynamics. For the quantum fields, the gauge fixing terms would be used to eliminate the redundant gauge degrees of freedom.

The action $S_{G F}^{\prime}$ contains the gauge fixing terms of quantum gauge and Goldstone fields. Normally, for the sake of convenience, we choose the Feynman-'t hooft gauge for the $S_{G F}^{\prime}$, which is given as

$$
S_{G F}^{\prime}=-\frac{1}{2}\left(D_{\mu} \widehat{W}^{\mu, a}+(v+\bar{H}) \tilde{\xi}^{a}\right)^{2} .
$$

By using the Euler-Lagrange equation for a field, the EOM of the classical gauge and Higgs bosons are determined as

$$
\begin{aligned}
D_{\mu}^{a b} \bar{W}^{b, \mu \nu} & =\frac{1}{4}(v+\bar{H})^{2} \bar{W}^{a, \nu}, \\
\partial^{2} \bar{H} & =\frac{1}{4}(v+\bar{H}) \bar{W} \cdot \bar{W}+\frac{\lambda}{4}(v+\bar{H})\left[(v+\bar{H})^{2}-v^{2}\right] .
\end{aligned}
$$


The EOM of the classical gauge bosons yields the following relation

$$
\partial_{\mu} \overline{H W}^{a, \mu}=-\frac{v+\bar{H}}{2} \partial \cdot \bar{W}^{a} .
$$

This relation is very important to eliminate all those terms with $\partial_{\mu} \overline{H W}^{a, \mu}$.

In order to give the proper definition to the propagator of the quantum Goldstone fields as in contrary to that given given in Eq. (1.2), we redefine the quantum Goldstone fields $\widetilde{\xi}$ as

$$
\widetilde{\xi} \rightarrow \frac{v}{v+\bar{H}} \widehat{\xi} .
$$

Then the partition function changes to

$$
\begin{aligned}
\mathcal{Z}\left[\bar{W}^{s}, \bar{H}\right]= & \int \mathcal{D} \widehat{W}_{\mu}^{a} \mathcal{D} \widehat{H} \mathcal{D} \widehat{\xi}^{b} \operatorname{det}\left\{\left(\frac{v+\bar{H}+\widehat{H}}{v+\bar{H}}\right) \delta(x-y)\right\} \\
& \exp \left(\mathcal{S}^{\prime}[\bar{W}, \bar{H} ; \widehat{W}, \widehat{H}, \widehat{\xi}]+S_{G F}\right) .
\end{aligned}
$$

And the gauge fixing term $S_{G F}$ is also modified in terms of $\widehat{W}_{\mu}^{a}$ and $\widehat{\xi}^{b}$. With this parameterization of the quantum Goldstone fields, we can define the propagator of Goldstone boson properly in either the coordinate or the momentum space. And no quartic divergence appears in the intermediate calculation steps. The quartic divergences are collected in the Eq. (4.8) as $\operatorname{det}\left\{\left(\frac{v+\bar{H}+\widehat{H}}{v+\bar{H}}\right) \delta(x-y)\right\}$. But if we parameterize the quantum Goldstone fields as given in [2], it becomes obscure to collect the quartic terms and verify the renormalizability of the theory.

The terms which are bilinear in quantum fields contribute to the one loop effective Lagrangian and can be presented in a standard form. However, these terms are obtained after a tedious calculation, manipulating the partial integrals and using the antisymmetric properties of $\mathrm{SU}(2)$ structure constants.

$$
\begin{aligned}
& \mathcal{L}_{\text {quadratic terms }}=-\frac{1}{2}\left(\widehat{W}^{a, \mu} \square_{\mu \nu}^{a b} \widehat{W}^{b, \nu}+\widehat{\xi}^{a} \square^{a b} \widehat{\xi}^{b}+\widehat{H} \square \widehat{H}+\right. \\
& \widehat{W}^{a, \mu}{ }_{X}^{\llcorner, a b} \widehat{\xi}^{b}+\widehat{\xi}^{a} \vec{X}_{\xi}^{\mu, a b} \widehat{W}^{b, \mu}+\widehat{W}^{a, \mu} \overleftarrow{X}_{H}^{\mu, a} \widehat{H}+ \\
& \left.\widehat{H} \vec{X}_{H}^{\mu, a} \widehat{W}^{a, \mu}+\widehat{\xi}^{a} \check{L}^{\llcorner a} \widehat{H}+\widehat{H} \vec{X}^{a} \widehat{\xi}^{a}\right)-\bar{c} \square_{\bar{c} c}^{a b} c,
\end{aligned}
$$

where the operators are defined as

$$
\begin{aligned}
\square_{\mu \nu}^{a b} & =\left[-(D \cdot D)^{a b}+m_{W}^{2} \delta^{a b}\right] g^{\mu \nu}+\sigma_{W}^{a b, \mu \nu}, \\
\square^{a b} & =\left[-(d \cdot d)^{a b}+m_{W}^{2} \delta^{a b}\right]+\sigma_{\xi}^{a b}, \\
\square & =\left[-\partial \cdot \partial+m_{H}^{2}\right]+\sigma_{H},
\end{aligned}
$$




$$
\begin{aligned}
& \square_{\bar{c} c}^{a b}=\left[-(D \cdot D)^{a b}+m_{W}^{2} \delta^{a b}\right]+\sigma_{c}^{a b}, \\
& \overleftarrow{X}_{\xi}^{\mu, a b}=g\left[-\frac{i}{2}(v+\bar{H}) \bar{W}_{\mu, G}^{a b}+\partial_{\mu} \bar{H} \delta^{a b}\right] \text {, } \\
& \vec{X}_{\xi}^{\mu, a b}=g\left[\frac{i}{2}(v+\bar{H}) \bar{W}_{\mu, G}^{a b}+\partial_{\mu} \bar{H} \delta^{a b}\right] \text {, } \\
& \overleftarrow{X}_{H}^{\mu, a}=\frac{g}{2}(v+\bar{H}) \bar{W}_{\mu}^{a}, \\
& \vec{X}_{H}^{\mu, a}=\frac{g}{2}(v+\bar{H}) \bar{W}_{\mu}^{a}, \\
& \check{\check{X}}^{a}=\check{\check{X}}^{\alpha, a} \partial^{\alpha}+\overleftarrow{X}_{0}^{a}, \\
& \vec{X}^{a}=\vec{X}^{\alpha, b} d^{b a, \alpha}+\vec{X}_{0}^{a}, \\
& \stackrel{\llcorner}{X}^{\alpha, a}=\bar{W}_{\alpha}^{a}, \\
& \check{X}_{0}^{a}=\frac{\partial \cdot \bar{W}^{a}}{2}, \\
& \vec{X}^{\alpha, b}=-\bar{W}_{\alpha}^{b}, \\
& \vec{X}_{0}^{a}=-\frac{\partial \cdot \bar{W}^{a}}{2},
\end{aligned}
$$

where the $\bar{W}_{\mu, G}^{a b}$ is defined as $\bar{W}_{\mu, G}^{a b}=\bar{W}_{\mu}^{e}\left(t^{e}\right)^{a b}$, and $t^{e}$ is the matrix of adjoint representation the group. The covariant differential operator $d_{\mu}$ of the Goldstone boson is defined as $d_{\mu}^{a b}=\partial_{\mu} \delta^{a b}-i g / 2 \bar{W}_{\mu, G}^{a b}$, while the covariant differential operator $D_{\mu}$ of the gauge boson is defined as $D_{\mu}^{a b}=\partial_{\mu} \delta^{a b}-i g \bar{W}_{\mu, G}^{a b}$. In the $S U(2)$ case, $\left(t^{e}\right)^{a b}=i f^{a e b}$, and $f^{a e b}$ is the structure constant of the $S U(2)$ Lie algebra. And the other quantities are defined as

$$
\begin{aligned}
\sigma_{W}^{a b, \mu \nu} & =\left[\frac{g^{2}}{4}(v+\bar{H})^{2}-\frac{g^{2}}{4} v^{2}\right] \delta^{a b} g^{\mu \nu}+2 i W^{a b, \mu \nu}, \\
\sigma_{\xi}^{a b} & =\left[\frac{g^{2}}{4}(v+\bar{H})^{2}-\frac{g^{2}}{4} v^{2}\right] \delta^{a b}-\frac{1}{4} \bar{W}_{G}^{a c} \cdot \bar{W}_{G}^{c b}+\frac{\partial^{2} \bar{H}}{v+\bar{H}} \delta^{a b}, \\
\sigma_{H} & =\frac{1}{4} \bar{W} \cdot \bar{W}+\frac{3 \lambda}{2} v \bar{H}+\frac{3 \lambda}{4} \bar{H}^{2}, \\
\sigma_{c}^{a b} & =\left[\frac{g^{2}}{4}(v+\bar{H})^{2}-\frac{g^{2}}{4} v^{2}\right] \delta^{a b} .
\end{aligned}
$$

Following the diagonalisation method prescribed in [2], we perform the Gaussian integral over quantum fields given by $\mathcal{L}_{\text {quadraticterms }}$ in Eq. (4.8). Hence we calculate the one loop effective generating functional $\Gamma_{1-\text { loop }}^{e f f}$, which is relevant up to $O\left(p^{4}\right)$

$$
\begin{aligned}
\Gamma_{1-\text { loop }}^{e f f}= & -\frac{1}{2} \operatorname{Tr}\left[\ln \square_{W}+\ln \square_{\xi}+\ln \square_{H}-2 \ln \square_{c}\right. \\
& -\vec{X}_{\xi} \square_{W}^{-1} \overleftarrow{X}_{\xi} \square_{\xi}^{-1}-\vec{X}_{H} \square_{W}^{-1} \overleftarrow{X}_{H} \square_{H}^{-1}-\vec{X} \square_{\xi}^{-1} \overleftarrow{X}_{H}^{-1} \square_{H}^{-1}
\end{aligned}
$$




$$
\begin{aligned}
& +\vec{X}_{H} \square_{W}^{-1} \overleftarrow{X}_{\xi} \square_{\xi}^{-1} \overleftarrow{X}^{-1}+\vec{X}_{H}^{-1} \vec{X}_{\xi} \square_{W}^{-1} \overleftarrow{X}_{H} \square_{H}^{-1} \\
& -\frac{1}{2} \vec{X}_{\xi} \square_{W}^{-1} \overleftarrow{X}_{\xi} \square_{\xi}^{-1} \vec{X}_{\xi} \square_{W}^{-1} \overleftarrow{X}_{\xi} \square_{\xi}^{-1}-\frac{1}{2} \vec{X}_{H} \square_{W}^{-1} \overleftarrow{X}_{H} \square_{H}^{-1} \vec{X}_{H} \square_{W}^{-1} \overleftarrow{X}_{H} \square_{H}^{-1} \\
& -\frac{1}{2} \vec{X} \square_{\xi}^{-1} \overleftarrow{X}_{H}^{-1} \vec{X}_{H}^{-1} \overleftarrow{X}_{\xi}^{-1}-\vec{X}_{H} \square_{W}^{-1} \overleftarrow{X}_{\xi} \square_{\xi}^{-1} \vec{X}_{\xi} \square_{W}^{-1} \overleftarrow{X}_{H} \square_{H}^{-1} \\
& -\vec{X} \square_{\xi}^{-1} \overleftarrow{X}_{\xi} \square_{W}^{-1} \vec{X}_{\xi} \square_{\xi}^{-1} \overleftarrow{X}^{-1} \square_{H}^{-1}-\vec{X}_{\xi}^{-1} \overleftarrow{X}^{\prime} \square_{H}^{-1} \vec{X}_{H} \square_{W}^{-1} \overleftarrow{X}_{H} \square_{H}^{-1} \\
& +\cdots] .
\end{aligned}
$$

Here the trace is over the points of the space-time, Lorentz and group indices. All these terms in the $\Gamma_{1-\text { loop }}^{e f f}$ can be expressed by a set of Feynman diagrams at the one-loop level. Although it may not be an one-to-one correspondence, but it guarantees the gauge invariance in each step of the calculation.

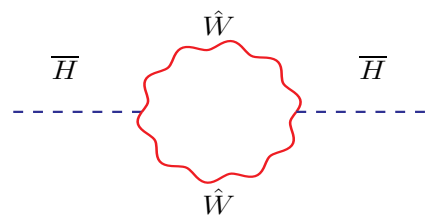

(a)

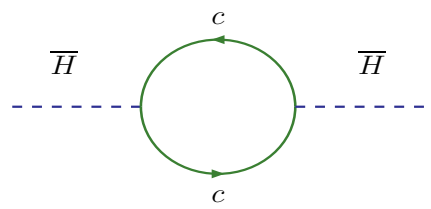

(d)

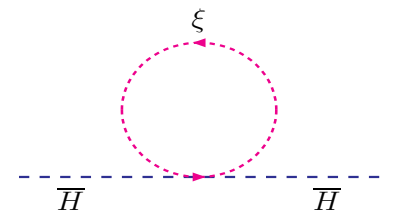

(g)

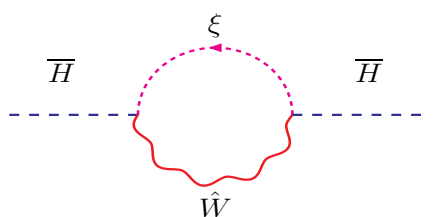

(b)

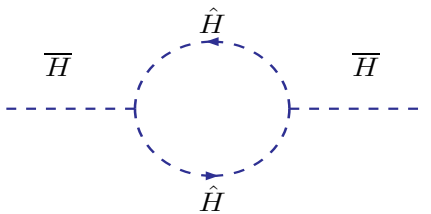

(e)

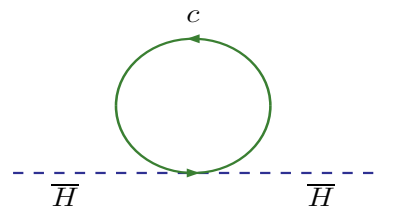

(h)

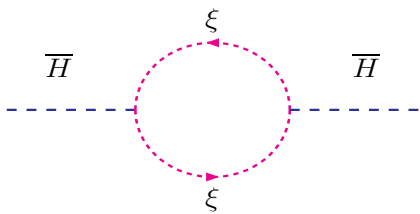

(c)

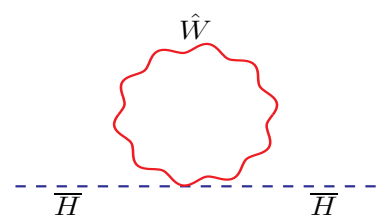

(f)

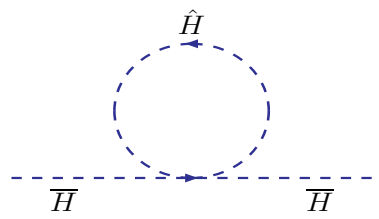

(i)

FIG. 1. Diagrams contributing to the $\delta m_{H}^{2}$ in the coordinate space calculation in $\overline{M S}$ scheme. In the decoupling limit only Figs. (c),(e) and (i) contributes to $\delta m_{H}^{2}$

By using the algebraic calculation we can skip the correspondence with Feynman diagrams at each step of calculation and just extract all the relevant terms that we are interested in, but we believe that it is always helpful to present linkage to the Feynman diagrams so as to make the comparison convenient and easy.

Considering the importance of the one loop correction to the Higgs boson mass, we only display the Feynman diagrams which contribute to this correction as shown in Fig. 
(1).

Next, we evaluate the one-loop effective generating functional $\Gamma_{1-\text { loop }}^{e f f}$ given in Eq. (4.12) in the coordinate space by using the heat kernel method and the covariant short distance expansion. The basics of the heat kernel method and the short distance expansion is provided in appendix A. The contributions of each term in $\Gamma_{1-\text { loop }}^{e f f}$ are listed in appendix B. With these formalism, the divergent terms in the one-loop effective Lagrangian are extracted and can be expressed as:

$$
\begin{aligned}
\mathcal{L}_{1-\text { loop }}^{\text {eff }}(\text { divergent })= & -\frac{1}{16 \pi^{2}} \frac{2}{\bar{\epsilon}}\left\{-\frac{43}{6} \frac{1}{4} \bar{W}_{\mu \nu}^{a} \bar{W}^{a \mu \nu}-\frac{3}{16} \frac{1}{4}(\bar{W} \cdot \bar{W})^{2}\right. \\
& +\frac{3}{8} \frac{\partial^{2} \bar{H}}{v+\bar{H}} \bar{W} \cdot \bar{W}-\frac{3}{4} \frac{\left(\partial^{2} \bar{H}\right)^{2}}{(v+\bar{H})^{2}} \\
& -\frac{9}{32} g^{2}(v+\bar{H})^{2} \bar{W} \cdot \bar{W}-\frac{3}{8} g^{2}(v+\bar{H}) \partial^{2} \bar{H} \\
& -\frac{3}{2} g^{2} \partial_{\mu} \bar{H} \partial^{\mu} \bar{H}-\frac{1}{64} \lambda^{2} v^{4}+\frac{3}{32} \lambda^{2}(v+\bar{H})^{2} v^{2} \\
& \left.-\frac{9}{64}\left(g^{4}+\lambda^{2}\right)(v+\bar{H})^{4}\right\}
\end{aligned}
$$

where the $2 / \bar{\epsilon}$ is defined as $2 / \epsilon-\ln (4 \pi)+\gamma_{E}$. Here we find there are extra divergent structures, like $(\bar{W} \cdot \bar{W})^{2}, \partial^{2} \overline{H W} \cdot \bar{W}$, and $\left(\partial^{2} \bar{H}\right)^{2}$, etc.

We now use the EOM for Higgs and gauge bosons to eliminate these extra divergent structures and get

$$
\begin{aligned}
\mathcal{L}_{1-\text { loop }}^{e f f}(\text { divergent })= & -\frac{1}{16 \pi^{2}} \frac{2}{\bar{\epsilon}}\left\{-\frac{43}{6} \frac{1}{4} \bar{W}_{\mu \nu}^{a} \overline{W^{a \mu \nu}}-\frac{3 g^{2}}{2} \partial \bar{H} \cdot \partial \bar{H}\right. \\
& -\frac{3 g^{2}}{8} \bar{W} \cdot \bar{W}(v+\bar{H})^{2}+\frac{3 \lambda}{32}\left(\lambda+2 g^{2}\right) v^{2}(v+\bar{H})^{2} \\
& \left.-\frac{3}{64}\left(3 g^{4}+2 \lambda g^{2}+4 \lambda^{2}\right)(v+\bar{H})^{4}-\frac{\lambda^{2}}{16} v^{4}\right\} .
\end{aligned}
$$

With the successful elimination of the extra divergent terms we re-establish the renormalizability of the theory which by itself provides the consistency check of our calculational strategy. There are couple of points worthy of remarks regarding the calculation of these divergent terms.

1. As a result of usage of EOM of the gauge bosons given in Eq. (4.6), there is no term $(\partial \cdot \bar{W})^{2}$ appearing in the divergent part given in Eq. (4.14) and hence it is no longer necessary to define the renormalization constant of the gauge parameter of the gauge fixing term of the classical gauge field. 
2. The use of EOM has its own ambiguities, e.g. consider the term, $(v+\bar{H}) \partial^{2} \bar{H}$. Here one can use the EOM of classical Higgs field either to eliminate $\partial^{2} \bar{H}$ or one can also replace the $(v+\bar{H}) \partial^{2} \bar{H}$ integral by $\partial_{\mu} \bar{H} \partial^{\mu} \bar{H}+v \partial^{2} H$. This ambiguity can be resolved by considering the fact that the operators $\partial \bar{H} \cdot \partial \bar{H} / 2$ and $\bar{W} \cdot \bar{W}(v+\bar{H})^{2} / 8$, in Eq. (2.7), essentially stems from $\left(D_{\mu} \phi\right)^{\dagger} \cdot D^{\mu} \phi$ in the linear form given in Eq. (2.2). Therefore, the renormalization constants of these two operators should always be same and this can be only achieved by using the EOM of Higgs boson.

3. We have performed our calculation using the Feynman-' $\mathrm{t}$ Hooft gauge $a=1$ and $\overline{M S}$ renormalization scheme in the coordinate space formalism. $\delta m_{H}^{2}$, in this formalism, at the decoupling limit can be expressed in terms of the scalar $B_{0}$ integrals as demonstrated in the appendix D.

$\delta m_{H}^{2}=\frac{g^{2}}{16 \pi^{2}} \frac{3 m_{H}^{2}}{8 m_{W}^{2}}\left\{m_{H}^{2} B_{0}\left(0, a m_{W}, a m_{W}\right)+3 m_{H}^{2} B_{0}\left(0, m_{H}, m_{H}\right)+A_{0}\left(m_{H}\right)\right\}$

Interpreting the Eq.(4.15) in the diagrammatic language, we find that at the decoupling limit, Fig. 1(b) contributes the term $B_{0}\left(0, m_{W}, m_{W}\right)$, Fig. 1(e) contributes the term $m_{H}^{2} B_{0}\left(0, m_{H}, m_{H}\right)$ and Fig. 1(i) contributes the term $A_{0}\left(m_{H}\right)$.

Performing the same calculation in the momentum space with Landau gauge $a=0$ and On-Shell renormalization scheme at the decoupling limit, yields

$$
\begin{aligned}
\left(\delta m_{H}^{2}\right)^{O n}= & \frac{g^{2}}{16 \pi^{2}} \frac{3\left(m_{H}^{2}\right)^{O n}}{8 m_{W}^{2}}\left\{\left(m_{H}^{2}\right)^{O n} \operatorname{Re}\left(B_{0}\left(\left.p^{2}\right|_{p^{2}=\left(m_{H}^{2}\right)^{O n}}, a m_{W}, a m_{W}\right)\right)\right. \\
& \left.+3\left(m_{H}^{2}\right)^{O n} B_{0}\left(\left.p^{2}\right|_{p^{2}=m_{H}^{2}}, m_{H}^{O n}, m_{H}^{O n}\right)+A_{0}\left(m_{H}^{O n}\right)\right\} .
\end{aligned}
$$

which is in complete agreement with the reference [2].

This would mean, the physical measurable quantities of the reference [2] can be retrieved at the decoupling limit, provided we make a proper correspondence of the coordinate space calculation in Feynman-'t Hooft gauge and $\overline{M S}$ renormalization scheme to the momentum space calculation in Landau gauge and On Shell renormalization scheme along with the input of the relation

$$
\left(m_{H}^{2}\right)^{\text {On }}=m_{H}^{2}+\left.\Sigma\left(p^{2}, m_{i}^{2}, m_{j}^{2}\right)\right|_{p^{2}=\left(m_{H}^{2}\right)^{O n}},
$$

where $\Sigma$ is the self energy corrections to the two point function of Higgs boson, the $p$ is the external momentum, and $m_{i}, m_{j}$ are the masses of particles in the loop. 
We proceed to construct explicitly the counter-terms from Eq. (4.14). The one-loop counter Lagrangian includes the terms which are linear in $\delta g, \delta v, \delta \mu^{2}$ and $\delta \lambda$, and can be expressed as

$$
\begin{aligned}
\delta \mathcal{L}^{\prime}= & \frac{\delta g}{2 g^{3}} W_{\mu \nu}^{a} W^{a \mu \nu}-\left\{\left(2 \delta v-\delta Z_{H} v\right)+\delta Z_{H}(v+\bar{H})\right\} \frac{(v+\bar{H})}{4} \operatorname{tr}\left[(D U)^{\dagger} \cdot(D U)\right] \\
& -\frac{1}{2} \delta Z_{H} \partial \bar{H} \cdot \partial \bar{H}-\frac{1}{2}\left(-2 \delta v+\delta Z_{H} v\right) \mu^{2}(v+\bar{H}) \\
& +\frac{1}{2}\left(\delta \mu^{2}+\mu^{2} \delta Z_{H}\right)(v+\bar{H})^{2}-\frac{\lambda}{8}\left(2 \delta v-\delta Z_{H} v\right)(v+\bar{H})^{3} \\
& -\frac{\delta \lambda+2 \delta Z_{H} \lambda}{16}(v+\bar{H})^{4},
\end{aligned}
$$

where $Z_{H}$ is the renormalization constant of Higgs field.

At the tree level, we can replace the the set of parameters of the theory such as $v, \mu^{2}, \lambda$ in terms of the another set of of parameters namely the tadpole parameter $t$, the gauge boson's mass $m_{W}$, and the Higgs boson's mass $m_{H}$ by the following equations

$$
\begin{aligned}
t & =\mu^{2} v-\frac{\lambda}{4} v^{3}, \\
m_{W}^{2} & =\frac{1}{4} g^{2} v^{2}, \\
m_{H}^{2} & =\frac{1}{2} \lambda v^{2} .
\end{aligned}
$$

And the corresponding relations of the counter terms between these two sets of parameters are given as

$$
\begin{aligned}
\frac{\delta v}{v} & =\frac{1}{2} \frac{\delta m_{W}^{2}}{m_{W}^{2}}-\frac{\delta g}{g}, \\
\frac{\delta \mu^{2}}{\mu^{2}} & =\frac{\delta m_{H}^{2}}{m_{H}^{2}}+\frac{3 g}{2 m_{W} m_{H}^{2}} \delta t, \\
\frac{\delta \lambda}{\lambda} & =\frac{\delta m_{H}^{2}}{m_{H}^{2}}-\frac{\delta m_{W}^{2}}{m_{W}^{2}}+2 \frac{\delta g}{g}+\frac{g}{2 m_{W} m_{H}^{2}} \delta t .
\end{aligned}
$$

Requiring combination of Eq. (4.14) and (4.18) to vanish, the counter terms at one loop level satisfy

$$
\begin{aligned}
\frac{\delta g}{g} & =g^{2} \frac{1}{16 \pi^{2}} \frac{1}{\bar{\epsilon}}\left\{-\frac{43}{12}\right\}, \\
\frac{\delta v}{v} & =\frac{1}{16 \pi^{2}} \frac{1}{\bar{\epsilon}}\left\{\frac{3}{2} g^{2}\right\}, \\
\frac{\delta \mu^{2}}{\mu^{2}} & =\frac{1}{16 \pi^{2}} \frac{1}{\bar{\epsilon}}\left\{\frac{3}{4}\left(\lambda-2 g^{2}\right)\right\}, \\
\frac{\delta \lambda}{\lambda} & =\frac{1}{16 \pi^{2}} \frac{1}{\bar{\epsilon}}\left\{\frac{3}{4}\left(3 \frac{g^{4}}{\lambda}-6 g^{2}+4 \lambda\right)\right\} .
\end{aligned}
$$


In addition to Eqs. (4.21a-4.21d), we also have $\delta Z_{H}=2 \delta v / v$, which guarantees that the vanishing of the divergences in the linear and trilinear interaction of the Higgs potential.

Then from Eqs. (4.20a-4.20c), the terms $\delta m_{W}^{2}, \delta m_{H}^{2}$ and $\delta t$ are determined as

$$
\begin{aligned}
\delta m_{W}^{2} & =2 m_{W}^{2}\left\{\frac{\delta v}{v}+\frac{\delta g}{g}\right\} \\
& =m_{W}^{2} \frac{1}{16 \pi^{2}} \frac{1}{\bar{\epsilon}} g^{2}\left\{-\frac{25}{6}\right\}, \\
\delta m_{H}^{2} & =\frac{m_{H}^{2}}{2}\left\{3 \frac{\delta \lambda}{\lambda}+6 \frac{\delta v}{v}-\frac{\delta \mu^{2}}{\mu^{2}}\right\} \\
& =m_{H}^{2} \frac{1}{16 \pi^{2}} \frac{1}{\bar{\epsilon}}\left\{-3 g^{2}+\frac{27}{4} \frac{g^{4}}{\lambda}+\frac{33}{4} \lambda\right\}, \\
\delta t & =\frac{\lambda \mu^{2}}{4}\left\{\frac{\delta \mu^{2}}{\mu^{2}}-\frac{\delta \lambda}{\lambda}-2 \frac{\delta v}{v}\right\} \\
& =\frac{9 \mu^{2}}{16} \frac{1}{16 \pi^{2}} \frac{1}{\bar{\epsilon}}\left\{g^{4}+\lambda^{2}\right\} .
\end{aligned}
$$

With those divergent terms given in Eq. (4.14) and the relations given in Eqs. (4.22a$4.22 \mathrm{c}$ ), it is straightforward to construct the renormalization constants in the modified minimal subtraction scheme and derive the corresponding running behavior of those parameters in the theory. However, the masses of gauge and Higgs boson given here are not the physical parameters measured in the experiments.

\section{THE COMPLETE ANOMALOUS COUPLINGS UP TO ONE-LOOP LEVEL}

From the one-loop effective Lagrangian given in Eq. (4.12) and the technique in the appendix B, we can also extract those finite terms which contribute to the trilinear and quartic anomalous interaction vertices. The finite part of the one-loop effective Lagrangian can be formulated as

$$
\begin{aligned}
\left.\mathcal{L}_{1-\text { loop }}^{\text {eff,full }} \text { (finite }\right)= & \delta t \bar{H}+\frac{\delta m_{W}^{2}}{2} W \cdot W+\frac{\delta m_{H}^{2}}{2} \bar{H}^{2}+\delta \lambda_{3} \bar{H}^{3} \\
& +\frac{\delta \lambda}{16} \bar{H}^{4}+\frac{\delta g}{2 g^{3}} W_{\mu \nu} W^{\mu \nu}+d_{1}^{f u l l} \operatorname{tr}\left[W_{\mu \nu} V^{\mu} V^{\nu}\right] \\
& +d_{2}^{f u l l} \operatorname{tr}\left[V_{\mu} V_{\nu}\right] \operatorname{tr}\left[V^{\mu} V^{\nu}\right]+d_{3}^{f u l l} \operatorname{tr}\left[V_{\mu} V^{\mu}\right] \operatorname{tr}\left[V_{\nu} V^{\nu}\right]+\cdots
\end{aligned}
$$

The dots represent higher order terms, like $(\bar{H} / v) W_{\mu \nu} W^{\mu \nu}$, etc.

The complete one-loop anomalous couplings of the vector boson sector in the renormalizable $\mathrm{SU}(2)$ theory can be divided into two parts: one is purely from the contribution of the gauge and the Goldstone bosons, the other is from the Higgs boson and its mixing 
with gauge and Goldstone bosons, i.e. $d_{i}^{f u l l}=d_{i}^{c, f u l l}+d_{i}^{h, f u l l}$. Although both the $d_{i}^{c, f u l l}$ and $d_{i}^{h, f u l l}$ are renormalization scale dependent, the combination of them indeed yield a renormalization scale independent results. The underlying reason for this is that the $S U(2)$ Higgs model is a renormalizable theory.

If we set the renormalization scale $\mu_{E}=m_{W}$, then $d_{i}^{c}$ become constant part, which is given as

$$
\begin{aligned}
& d_{1}^{c, \text { full }}=\frac{1}{16 \pi^{2}}\left(-\frac{11}{6}\right) \\
& d_{2}^{c, \text { full }}=\frac{1}{16 \pi^{2}}\left(\frac{1}{2}\right) \\
& d_{3}^{c, \text { full }}=\frac{1}{16 \pi^{2}}\left(-\frac{1}{6}\right)
\end{aligned}
$$

The Higgs boson dependent terms of anomalous couplings are given as

$$
\begin{aligned}
& d_{1}^{h, \text { full }}=\frac{1}{16 \pi^{2}}\left\{\frac{-197+154 r-17 r^{2}}{72(-1+r)^{2}}+\frac{r\left(36-27 r+r^{2}\right) \ln (r)}{12(-1+r)^{3}}\right\}, \\
& d_{2}^{h, \text { full }}=\frac{1}{16 \pi^{2}}\left\{\frac{-71+208 r-17 r^{2}}{72(-1+r)^{2}}+\frac{r^{2}(-21+r) \ln (r)}{12(-1+r)^{3}}\right\}, \\
& d_{3}^{h, \text { full }}=\frac{1}{16 \pi^{2}}\left\{\frac{13-374 r+13 r^{2}}{144(-1+r)^{2}}+\frac{\left(27+3 r+87 r^{2}-r^{3}\right) \ln (r)}{48(-1+r)^{3}}\right\},
\end{aligned}
$$

where $r=m_{H}^{2} / m_{W}^{2}$.

The anomalous couplings given in Eqs. (5.3a-5.3c) might be infrared singular at their appearance when $r$ approaches to 1, but we find that this is not true, actually the infrared singularities from the non-log and log parts just cancel exactly with each other. In the limit with $r$ approaches to 1 , we have

$$
\begin{aligned}
& d_{1}^{h, \text { full }}=\frac{1}{16 \pi^{2}}\left(-\frac{4}{3}\right), \\
& d_{2}^{h, \text { full }}=\frac{1}{16 \pi^{2}}\left(-\frac{2}{3}\right), \\
& d_{3}^{h, \text { full }}=\frac{1}{16 \pi^{2}}\left(\frac{5}{6}\right) .
\end{aligned}
$$

In the limit with $r \rightarrow \infty$, the non-decoupling parts of these $d_{i}^{h}$ are given as

$$
\begin{aligned}
& d_{1}^{h, \text { full }}=\frac{1}{16 \pi^{2}}\left\{-\frac{17}{72}+\frac{1}{12} \ln (r)\right\}, \\
& d_{2}^{h, \text { full }}=\frac{1}{16 \pi^{2}}\left\{-\frac{17}{72}+\frac{1}{12} \ln (r)\right\}, \\
& d_{3}^{h, \text { full }}=\frac{1}{16 \pi^{2}}\left\{\frac{13}{144}-\frac{1}{48} \ln (r)\right\} .
\end{aligned}
$$


In order to derive the one-loop matching conditions, we should also perform the one-loop calculation for the non-renormalizable effective chiral $S U(2)$ theory. After performing the path integral as prescribed in reference [9] and appendix $\mathrm{C}$, we construct the counter terms to eliminate the divergences. Then we formulate the finite one-loop effective Lagrangian as

$$
\begin{aligned}
\mathcal{L}_{1-\text { loop }}^{\text {eff,chl }}(\text { finite })= & \frac{\delta m_{W}^{2}}{2} W \cdot W+\frac{\delta g}{2 g^{3}} W_{\mu \nu} W^{\mu \nu}+d_{1}^{e f f} \operatorname{tr}\left[W_{\mu \nu} V^{\mu} V^{\nu}\right] \\
& +d_{2}^{\text {eff }} \operatorname{tr}\left[V_{\mu} V_{\nu}\right] \operatorname{tr}\left[V^{\mu} V^{\nu}\right]+d_{3}^{\text {eff }} \operatorname{tr}\left[V_{\mu} V^{\mu}\right] \operatorname{tr}\left[V_{\nu} V^{\nu}\right]+\cdots .
\end{aligned}
$$

As in the full theory, $d_{i}^{\text {eff }}$ can also be decomposed into two parts: the part from the contribution of Goldstone and vector bosons, $d_{i}^{c, e f f}$, and the part dependent on the Higgs boson's mass, $d_{i}^{h, e f f}$. We perform the similar computational steps with the $\mathrm{SU}(2)$ chiral Lagrangian given in Eq. (3.1a) to extract the anomalous couplings $d_{i}^{\text {eff }}$. The contribution of gauge and Goldstone bosons is given as (here we have substituted the renormalization scale $\left.\mu_{E}=m_{H}\right)$ :

$$
\begin{aligned}
& d_{1}^{c, e f f}=\frac{1}{16 \pi^{2}}\left(-\frac{11}{6}+\frac{1}{12} \ln (r)\right), \\
& d_{2}^{c, \text { eff }}=\frac{1}{16 \pi^{2}}\left(\frac{1}{2}+\frac{1}{12} \ln (r)\right), \\
& d_{3}^{c, \text { eff }}=\frac{1}{16 \pi^{2}}\left(\frac{1}{6}+\frac{1}{24} \ln (r)\right) .
\end{aligned}
$$

In order to compute the effective Lagrangian (5.6) up to the one-loop level, we require the tree level matching conditions as input. According to the tree-level matching conditions, which are presented later in Eqs. (6.3a-6.3c), we know that the terms dependent on the Higgs boson's mass are those which are dependent on $d_{3}^{\text {tree }}$, so we only retain terms proportional to $d_{3}^{\text {tree }}$ and its powers. Then we have the one-loop anomalous couplings which are given as

$$
\begin{aligned}
& d_{1}^{h, e f f}=\frac{1}{16 \pi^{2}}\left(d_{3}^{\text {tree }} g^{2}-5 d_{3}^{\text {tree }} g^{2} \ln (r)\right), \\
& d_{2}^{h, e f f}=\frac{1}{16 \pi^{2}}\left(\frac{3}{2}\left(d_{3}^{\text {tree }}\right)^{2} g^{4}+\left(d_{3}^{\text {tree }}\right)^{2} g^{4} \ln (r)-d_{3}^{\text {tree }} g^{2} \ln (r)\right), \\
& d_{3}^{h, e f f}=\frac{1}{16 \pi^{2}}\left(-\frac{39}{4}\left(d_{3}^{\text {tree }}\right)^{2} g^{4}+6 d_{3}^{\text {tree }} g^{2}+\frac{25}{2}\left(d_{3}^{\text {tree }}\right)^{2} g^{4} \ln (r)-6 d_{3}^{\text {tree }} g^{2} \ln (r)\right) .
\end{aligned}
$$

\section{THE TREE LEVEL AND THE ONE-LOOP MATCHING CONDITIONS}

According to the standard matching procedure specified in [1], we should match the full theory and the effective theory order by order. The effective couplings are organized in term of loops as 


$$
d_{i}=d_{i}^{\text {tree }}+d_{i}^{1-l o o p}+\ldots .
$$

To determine the higher order of couplings, we need to know the lower order ones.

In the $S U(2)$ Higgs model case, at the tree level, it suffices to integrate out the Higgs boson, using the EOM by assuming that $\partial^{2} \bar{H}$ is negligibly small, which then expresses the Higgs boson in terms of the low energy dynamical degrees of freedom and can be formulated as

$$
\bar{H}=\frac{v}{2 m_{H}^{2}}(D U)^{\dagger} \cdot(D U)+\cdots,
$$

The omitted terms contain at least four covariant partials and belong to the higher order operators.

To set up the matching conditions at the tree level we first use Eq. (6.2) to eliminate the Higgs field in the modified Lagrangian given in Eq. (2.12), at the matching scale ( which is always taken at the scalar mass $\mu_{E}=m_{H}$ ). Then we compare the terms of this resulting Lagrangian with the effective Lagrangian given in Eq. (3.1c). Thus the matching conditions for the effective couplings at the tree level are determined as

$$
\begin{aligned}
& d_{1}^{\text {tree }}\left(m_{H}\right)=0, \\
& d_{2}^{\text {tree }}\left(m_{H}\right)=0, \\
& d_{3}^{\text {tree }}\left(m_{H}\right)=\frac{v^{2}}{8 m_{H}^{2}}=\frac{m_{W}^{2}}{2 g^{2} m_{H}^{2}}, \cdots .
\end{aligned}
$$

In its decoupling limit $m_{H} \rightarrow \infty(\lambda \rightarrow \infty)$, all these three effective couplings vanish. Generally, for certain theoretical reasons (say, the validity of perturbation theory ) $\lambda$ does not tend to $\infty$, and is usually considered to be of the $O(1)$, as we realize in the case of standard model. So, $d_{3}^{\text {tree }}$ can be quite large compared with other anomalous couplings. However, in technicolor theories, all of these anomalous couplings might be quite large.

To derive the matching conditions at one-loop level, at the first step, both the calculations in the effective as well as in the full theory are to be considered up to the one-loop order. As standard in perturbation theory, we would require the tree level results of $d_{i}^{\text {tree }}\left(m_{H}\right)$ as inputs to compute the higher order results $d_{i}^{1-l o o p}\left(m_{H}\right)$. Then, at the second step, while matching the one-loop effective Lagrangian of these two theories, we eliminate Higgs field using EOM given in (6.2) as specified in Eq. (1.1). Following these two steps and using the one-loop anomalous couplings of the full theory in Eqs. (5.2a-5.2c) and (5.3a-5.3c) and those in the effective theory in Eqs. (5.7a-5.7c) and (5.8a-5.8c), we arrive at the following matching conditions. 


$$
\begin{aligned}
d_{1}^{1-\text { loop }}\left(m_{H}\right)= & \frac{1}{16 \pi^{2}}\left\{\frac{-\left(36+125 r-118 r^{2}+17 r^{3}\right)}{72(-1+r)^{2} r}\right. \\
& \left.-\frac{\left(30-91 r+57 r^{2}-6 r^{3}\right) \ln (r)}{12(-1+r)^{3} r}\right\}, \\
d_{2}^{1-\text { loop }}\left(m_{H}\right)= & \frac{1}{16 \pi^{2}}\left\{-\frac{27-54 r+98 r^{2}-208 r^{3}+17 r^{4}}{72(-1+r)^{2} r^{2}}\right. \\
& \left.-\frac{\left(-3+15 r-28 r^{2}+24 r^{3}+12 r^{4}\right) \ln (r)}{12(-1+r)^{3} r^{2}}\right\}, \\
d_{3}^{1-\text { loop }}\left(m_{H}\right)= & \frac{1}{16 \pi^{2}}\left\{\frac{1458-3150 r+1763 r^{2}-1018 r^{3}+107 r^{4}}{288(-1+r)^{2} r^{2}}\right. \\
& \left.-\frac{\left(120-318 r+145 r^{2}+69 r^{3}-156 r^{4}\right) \ln (r)}{48(-1+r)^{3} r^{2}}\right\} .
\end{aligned}
$$

Here it is worth mentioning that two operators, $H^{2}$ and $H \bar{W} \cdot \bar{W}$, in the one-loop effective generating functional of the full theory also contributes to $d_{3}^{1-l o o p}\left(m_{H}\right)$.

In the limit with $r \rightarrow \infty$, the non-decoupling parts of these $d_{i}^{1-\text { loop }}$ are given as

$$
\begin{aligned}
& d_{1}^{1-\text { loop }}\left(m_{H}\right)=\frac{1}{16 \pi^{2}}\left(-\frac{17}{72}\right), \\
& d_{2}^{1-\text { loop }}\left(m_{H}\right)=\frac{1}{16 \pi^{2}}\left(-\frac{17}{72}\right), \\
& d_{3}^{1-\text { loop }}\left(m_{H}\right)=\frac{1}{16 \pi^{2}}\left(\frac{107}{288}\right) .
\end{aligned}
$$

From Eqs. (6.5a-6.5c), we find that at the decoupling limit, the matching conditions shred off their dependencies on the non-decoupling logarithms. These constants, when compared with those obtained in [2], are same for the first two anomalous couplings while it appears to be different for the $d_{3}^{1-l o o p}\left(m_{H}\right)$. This reason for this difference can be traced back in the difference arising in $\delta m_{H}^{2}$ given in Eq.(4.15) and Eq. (4.16) and their evaluation as given in appendix D. The $p^{2}$ dependence in the scalar $B_{0}$ integrals affects the finite part of $\delta m_{H}^{2}$, and manifests its bearing in the evaluation of $d_{3}^{1-l o o p}$ when the Higgs field is integrated out. Although $d_{3}^{1-l o o p}$ given here is different from that in [2], the total $d_{3}=d_{3}^{\text {tree }}+d_{3}^{1-\text { loop }}$, which is the physical and detectable quantity in experiments, is just the same for both renormalization schemes when the relation of the Higgs boson's mass between them given in Eq. (4.17) is used. 


\section{COMPARISON WITH THE RENORMALIZATION GROUP EQUATION METHOD}

The RGE of an effective theory is one of the its basic ingredients, and its function is to sum over quantum corrections in leading log (one-loop RGEs), next leading log (two-loop RGEs), and so on. For instance, the one-loop RGEs can sum over all leading log of all loop diagrams. However, it differs from the direct loop calculations, where the radiative contribution are computed and organized by loops.

In order to check the reliability of the effective field theory method, it is constructive to compare the predictions of the one-loop direct calculation and those of the one-loop RGEs. The one-loop RGEs of the chiral effective SU(2) Lagrangian can be derived by computing the one-loop irreducible vertex generating functional, and has been computed in the reference [9]. Appendix $\mathrm{C}$ outlines this calculation with the corrections to reference [9]. These corrected RGEs can be tabulated as

$$
\begin{aligned}
\frac{d g^{2}}{d t}= & \frac{g^{4}}{8 \pi^{2}}\left[-\frac{29}{4}-\frac{20 d_{1} g^{2}}{3}-\frac{23 d_{1}^{2} g^{4}}{24}\right] \\
\frac{d v}{d t}= & \frac{v}{8 \pi^{2}}\left[\frac{3 g^{2}}{2}+\left(5 d_{1}-10 d_{2}-\frac{35 d_{3}}{2}\right) g^{4}+\frac{13 d_{1}^{2} g^{6}}{8}\right] \\
\frac{d d_{1}}{d t}= & \frac{1}{8 \pi^{2}}\left\{-\frac{1}{12}+\left(-\frac{26 d_{1}}{3}-\frac{5 d_{2}}{2}+5 d_{3}\right) g^{2}\right. \\
& \left.-\frac{109 d_{1}^{2} g^{4}}{12}-\frac{19 d_{1}^{3} g^{6}}{12}\right\} \\
\frac{d d_{2}}{d t}= & \frac{1}{8 \pi^{2}}\left\{-\frac{1}{12}+\left(\frac{d_{1}}{2}+3 d_{2}+d_{3}\right) g^{2}\right. \\
& +\left[\frac{87 d_{1}^{2}}{32}-6 d_{2}^{2}-5 d_{2} d_{3}-d_{3}^{2}+d_{1}\left(5 d_{2}-4 d_{3}\right)\right] g^{4} \\
& +\left[-d_{1}^{3}+d_{1}^{2}\left(-\frac{5}{4} d_{2}-\frac{5 d_{3}}{4}\right)\right] g^{6} \\
& \left.-\frac{43 d_{1}^{4} g^{8}}{24}\right\}, \\
& \frac{1}{8 \pi^{2}}\left\{-\frac{1}{24}+\left(\frac{5 d_{2}}{2}+6 d_{3}\right) g^{2}\right. \\
+ & {\left[-\frac{155 d_{1}^{2}}{32}-\frac{9 d_{2}{ }^{2}}{4}+d_{1}\left(10 d_{2}+28 d_{3}\right)-13 d_{2} d_{3}-\frac{25 d_{3}{ }^{2}}{2}\right] g^{4} } \\
+ & {\left.\left[\frac{-21 d_{1}^{3}}{4}+d_{1}^{2}\left(\frac{71 d_{2}}{8}-16 d_{3}\right)\right] g^{6}-\frac{19 d_{1}{ }^{4} g^{8}}{12}\right\} }
\end{aligned}
$$


where $t$ is defined as $t=\ln \left(\mu_{E}\right)$. Here we have utilized the modified momentum power counting rule for the anomalous couplings [9], in which the momentum dimension of $d_{i}$ is set to be -2 , so that the momentum dimension $d_{i} g^{2}$ is zero. Therefore, the terms containing $d_{i} g^{2}$ are treated similarly as those of the constants in the $\beta$ functions.

In order to compare and contrast, we formulate the results of the direct integrating-out method [2] with the decoupling limit in its RGE form, which read

$$
\begin{aligned}
\frac{d g^{2}}{d t} & =\frac{g^{4}}{8 \pi^{2}}\left\{-\frac{29}{4}\right\}, \\
\frac{d v}{d t} & =\frac{v}{8 \pi^{2}}\left\{\frac{3 g^{2}}{2}\right\}, \\
\frac{d d_{1}}{d t} & =\frac{1}{8 \pi^{2}}\left\{-\frac{1}{12}\right\}, \\
\frac{d d_{2}}{d t} & =\frac{1}{8 \pi^{2}}\left\{-\frac{1}{12}\right\}, \\
\frac{d d_{3}}{d t} & =\frac{1}{8 \pi^{2}}\left\{-\frac{1}{24}\right\} .
\end{aligned}
$$

To retrieve Eqs. (7.2a-7.2e) from Eqs. (7.1a -7.1e), we consider the case where $d_{i}$ 's are assumed to be of the order of $1 /(4 \pi)^{2}$. Substituting these $d_{i}$ 's in right hand side of Eqs. (7.1a-7.1e), we find that these terms are smaller than the leading constant terms and thus they would correspond to the two loop effects. So, keeping the relevance of our calculation

up to one loop order, we neglect these terms from higher order loops and hence reproduce same set of equations as given in (7.2a-7.2e).

\section{NUMERICAL ANALYSIS}

For the numerical analysis, we mimic the standard model by choosing the mass of gauge boson $m_{W}$ to be $80 \mathrm{GeV}$. The Higgs boson is assumed to be heavier than the gauge bosons $W$. The initial condition for the coupling $g$ and the vacuum expectation value $v$ is fixed at the lower boundary point, $\mu_{E}=m_{W}$. The coupling $g\left(m_{W}\right)$ is chosen to satisfy

$$
\alpha_{g}=\frac{g^{2}}{4 \pi}=\frac{1}{30}
$$

which gives $g\left(m_{W}\right)=0.65$ and the vacuum expectation value is then fixed by $m_{W}=\frac{1}{2} g v$, which gives $v\left(m_{W}\right)=247$. The initial conditions for $d_{i}$ 's $i=1,2,3$ are chosen to be fixed at the matching scale, $\mu_{E}=m_{H}$, as given in Eqs. (6.3a-6.3c) and Eqs. (6.4a-6.4c), both at the tree and the one-loop level. 
It is important to clarify that the masses of gauge boson and Higgs boson in the $\overline{M S}$ and on-shell renormalization schemes are different. The relation of the masses of the gauge boson between the $\overline{M S}$ and the on-shell renormalization scheme up to one-loop level in the full theory is given as

$$
m_{M, \text { on-shell }}^{2}=m_{W}^{2}\left[1+\frac{1}{16 \pi^{2}}\left(\frac{5 r-3}{16}+\frac{r(5-2 r)}{8(r-1)} \ln (r)-\frac{r+3}{4} \ln \left(r_{w}\right)\right)\right]
$$

where $r_{W}=m_{W}^{2} / \mu_{E}^{2}$. However the perturbation theory breaks down for very heavy Higgs boson and large $r$. Therefore, for the sake of simplicity, in the numerical analysis, we will only consider the tree level relations between the masses of these two schemes, and assume that the gauge boson's mass is the one which is measured in the experiments.

We consider three cases to show the effects of matching conditions. The first case is just the tree-level matching conditions, the second case is the one-loop matching conditions in the decoupling limit, and the third one is the exact one-loop matching conditions.

In the Figs. 2 , 3 and 4 we plot the magnitude of the effective couplings $d_{i}$ 's with varying mass of the Higgs boson for these three different cases.

Figs. 2(a), 3(a) and 4(a) indicate that for most range of Higgs mass, the constant contribution from the gauge and Goldstone bosons is numerically larger than the contribution from the part dependent on Higgs boson mass.

If only considering the non-decoupling log terms alone, Fig. 2(b) shows that the direct integrating-out method is worse than the RGE method when Higgs boson is relatively light. Fig. 2(c) establishes the fact that after taking into account the one-loop nondecoupling constants at the matching scale, both the methods improve. However, the RGE method comes out to be better than the direct integrating-out method. Fig. 2(d) confirms that after using the exact one-loop matching condition, the RGE method gives better prediction than the direct integrating-out method for a wide range of Higgs boson's mass.

Fig. 3(b) shows that with the tree level matching conditions, both the RGE method and the direct integrating method deviate from the exact one-loop result significantly. Fig. 3(c) shows that when taking into account the one-loop non-decoupling constants, predictions of both methods improve. Fig. 3(d) shows when the exact one-loop matching conditions are used, for a wide range of Higgs boson the RGE method is better than the direct integrating-out method.

Figs. 2(c), 2(d), 3(c) and Fig. 3(d) also show that if Higgs boson's mass becomes heavy, the predictions of the direct integrating-out method, the RGE method, and the exact oneloop calculation converge. While when Higgs boson's mass becomes light, the difference 
between the effective field theory method and the exact one-loop calculation become large, which indicates the effects of higher order operators in the effective Lagrangian.

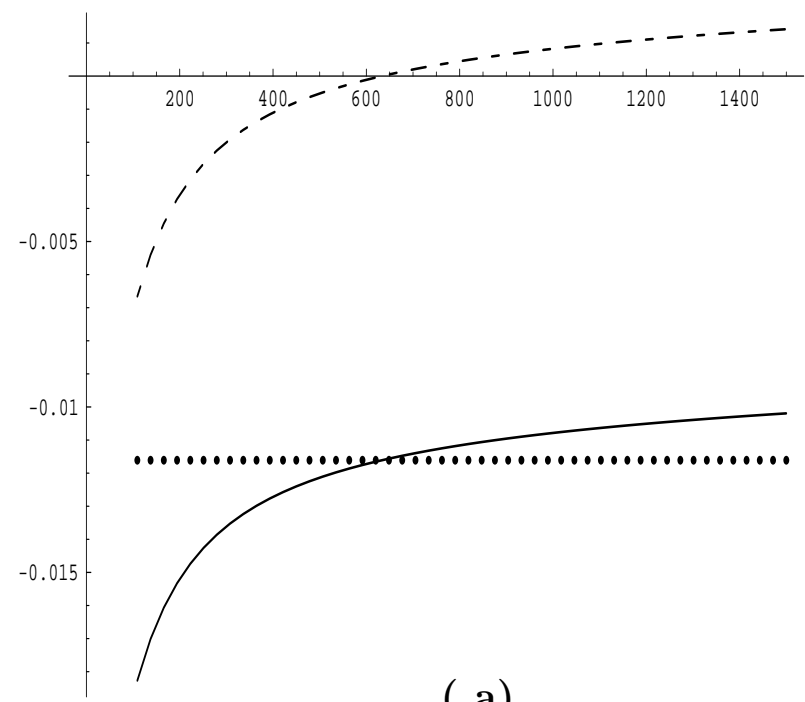

( a)

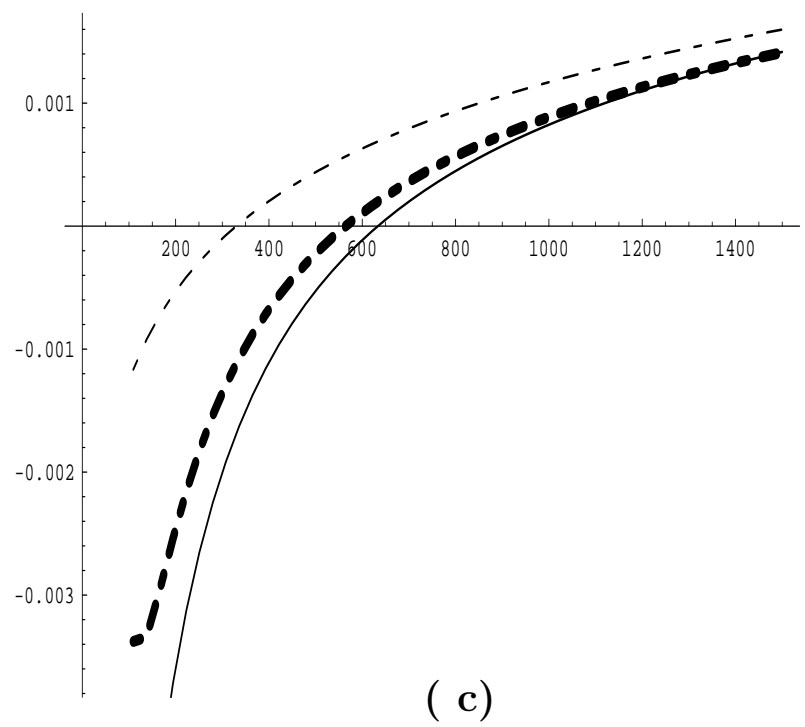

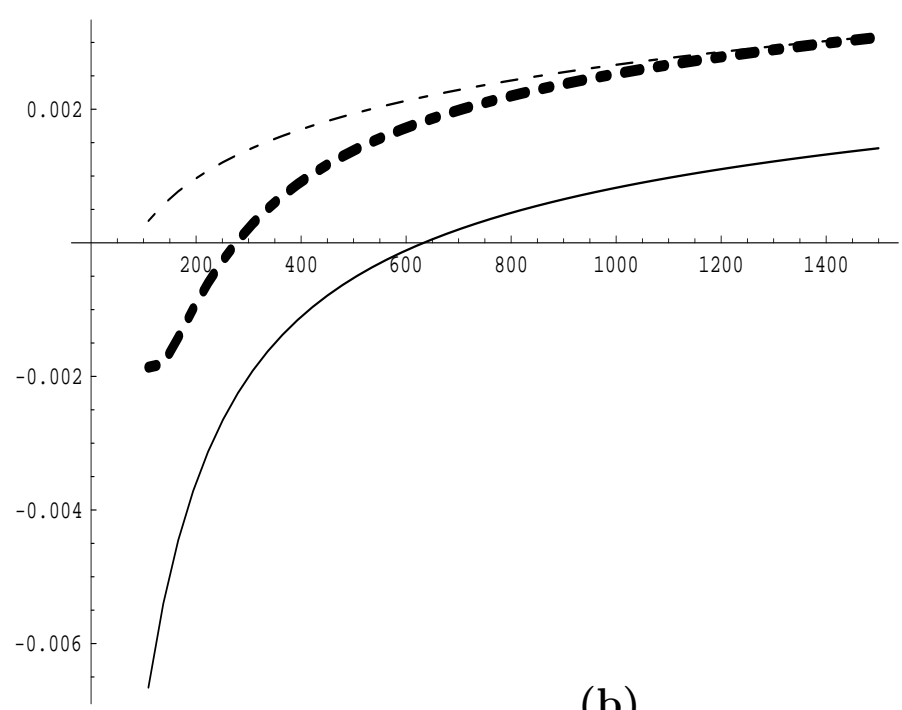

(b)

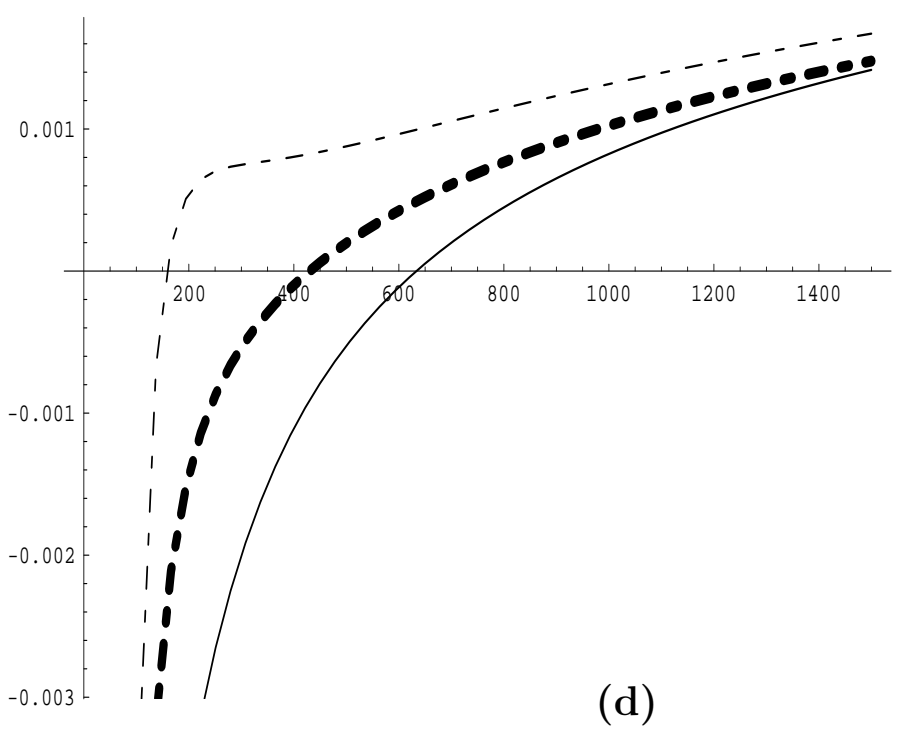

FIG. 2. Variation of $d_{1}\left(m_{W}\right)$ in $y$ axis is shown with the $m_{H}$ (in $G e V$ ) in $x$ axis. Figure (a) shows the constant part (dotted line), $m_{H}$ dependent part (dashed line) and the sum of these two (solid line) from the 1-loop contribution. Figures (b), (c), and (d) corresponds to tree level, 1-loop in the decoupling limit and exact 1-loop matching conditions respectively, and each of them depict the comparison between the direct integrating-out method (thin dashed line), the RGE method in the effective theory (thick dashed line) and 1-loop in the full theory (solid line). 


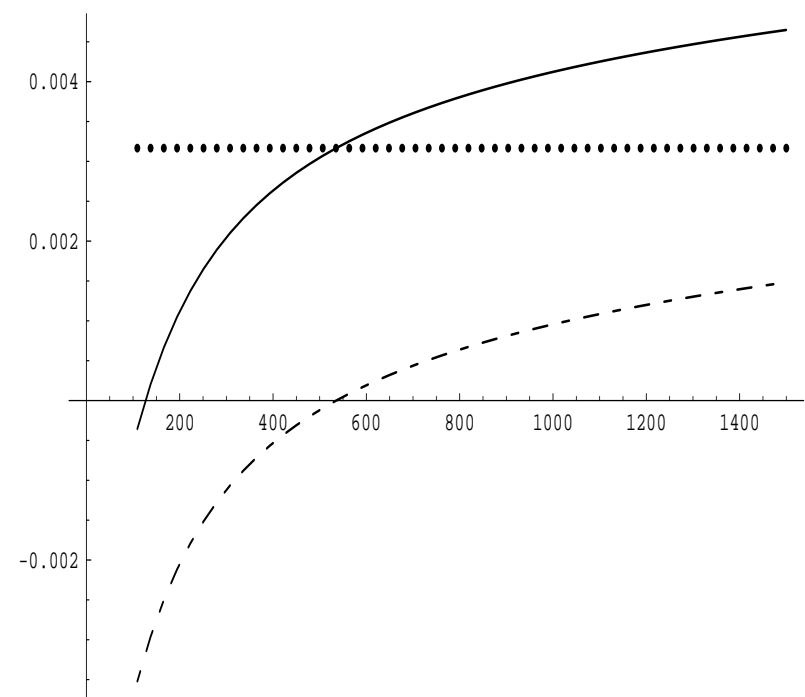

( a)

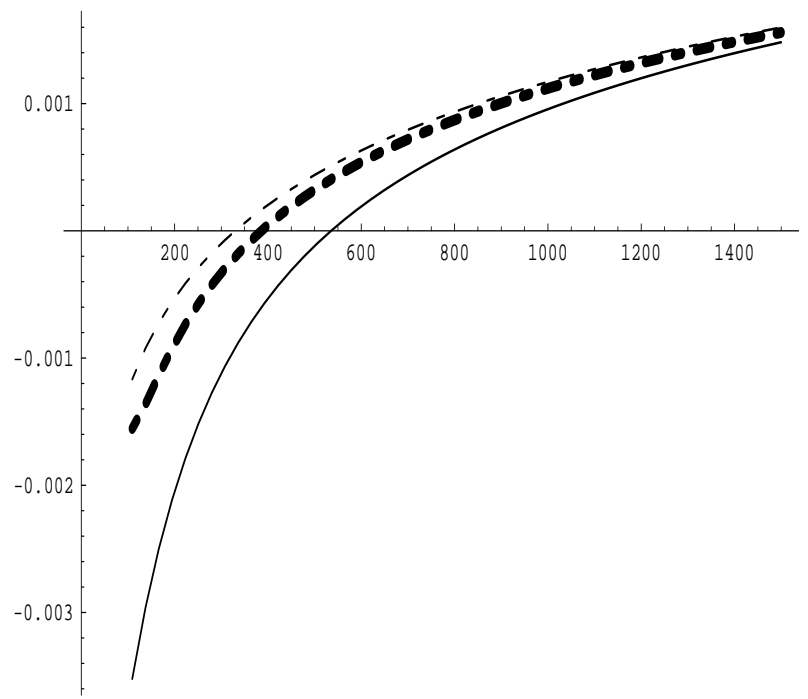

( c)

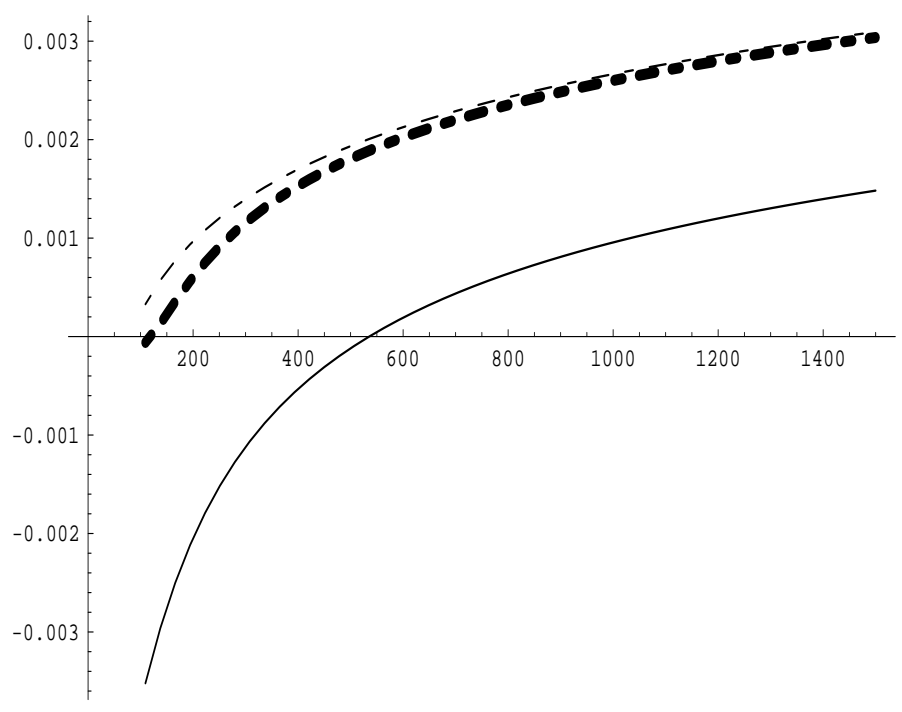

(b)

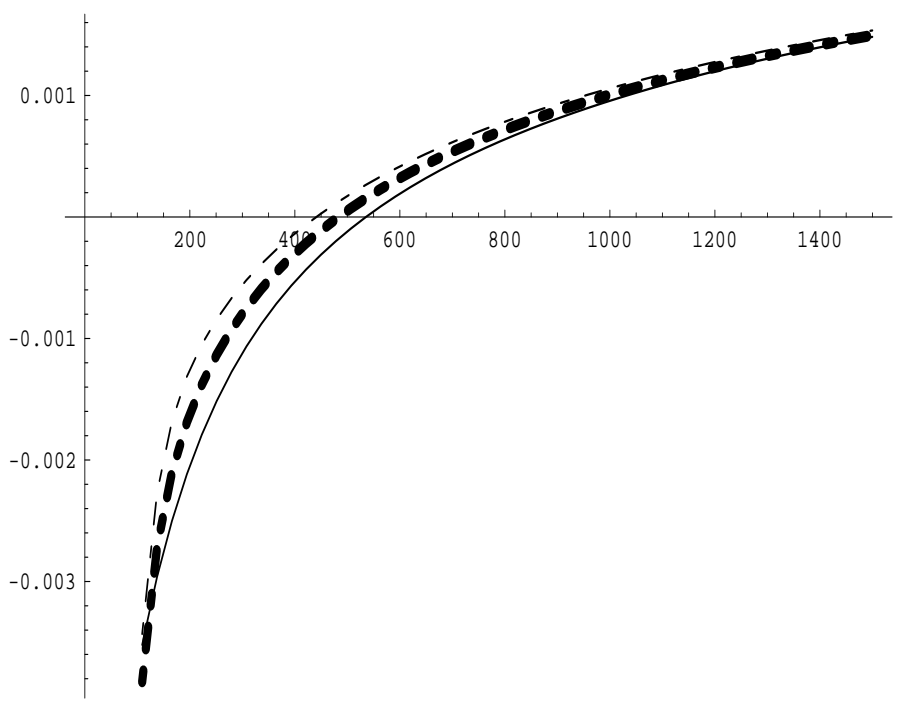

(d)

FIG. 3. Variation of $d_{2}\left(m_{W}\right)$ in $y$ axis is shown with the $m_{H}$ (in $G e V$ ) in $x$ axis. Figure (a) shows the constant part (dotted line), $m_{H}$ dependent part (dashed line) and the sum of these two (solid line) from the 1-loop contribution. Figures (b), (c), and (d) corresponds to tree level, 1-loop in the decoupling limit and exact 1-loop matching conditions respectively, and each of them depict the comparison between the direct integrating-out method (thin dashed line), the $R G E$ method in the effective theory (thick dashed line) and 1-loop in the full theory (solid line). 


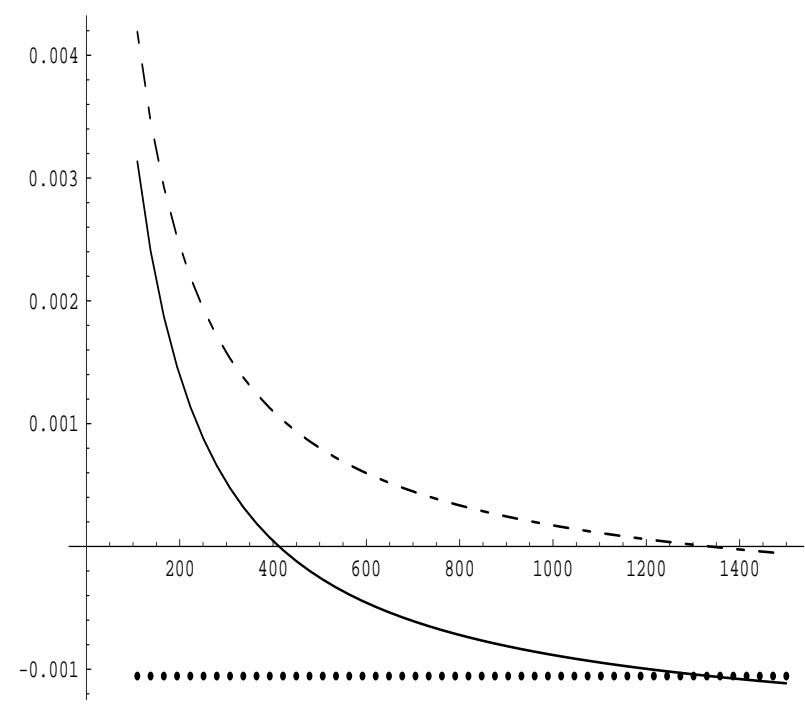

( a)

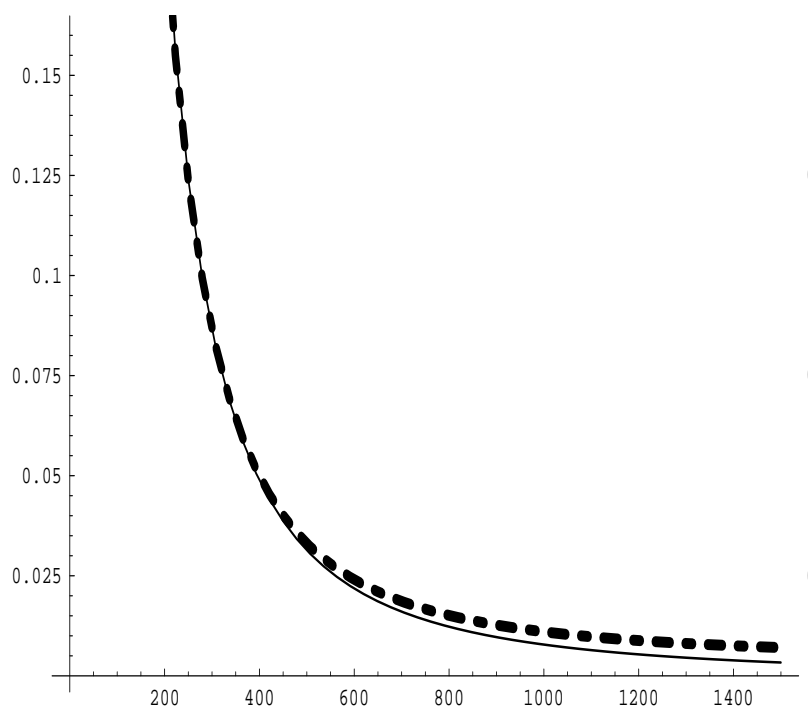

( c)

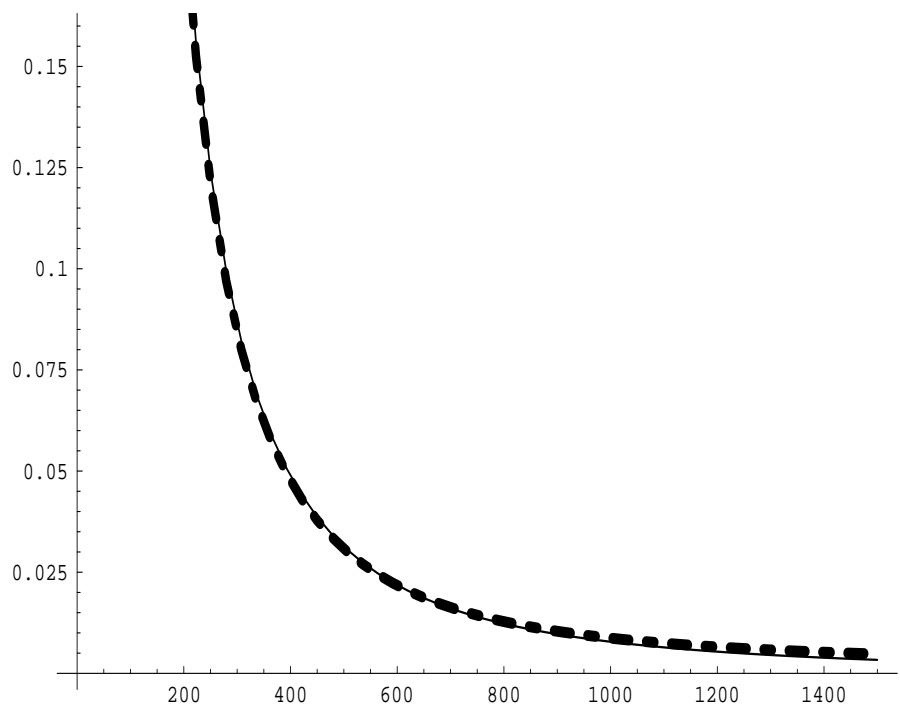

(b)

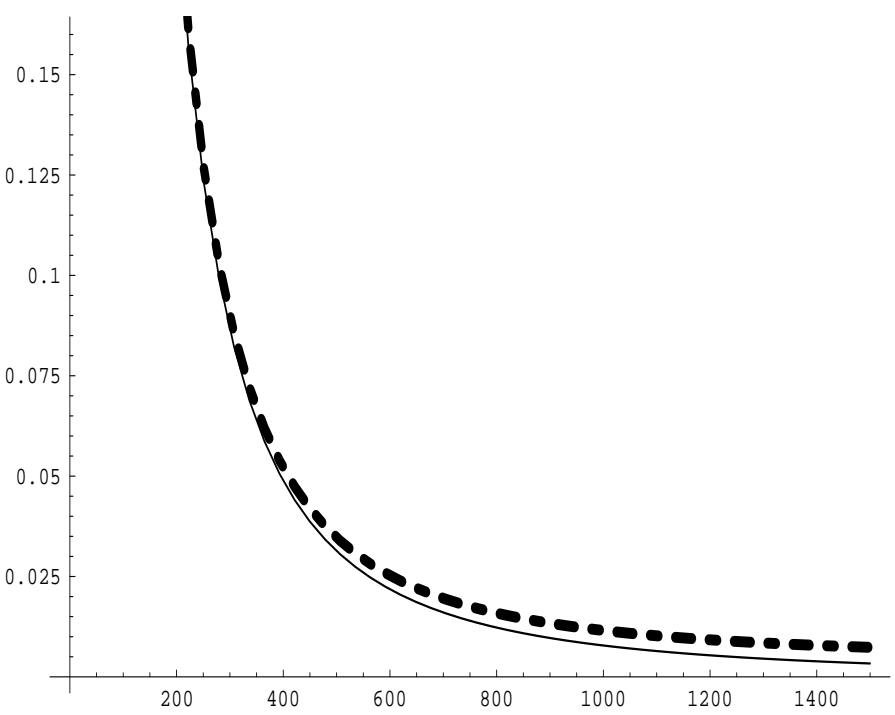

(d)

FIG. 4. Variation of $d_{3}\left(m_{W}\right)$ in $y$ axis is shown with the $m_{H}$ (in $G e V$ ) in $x$ axis. Figure (a) shows the constant part (dotted line), $m_{H}$ dependent part (dashed line) and the sum of these two (solid line) from the 1-loop contribution. Figures (b), (c), and (d) corresponds to tree level, 1-loop in the decoupling limit and exact 1-loop matching conditions respectively, and each of them depict the comparison between the direct integrating-out method (thin dashed line), the $R G E$ method in the effective theory (thick dashed line) and 1-loop in the full theory (solid line).

The tree level contribution of $d_{3}$ is overwhelming against the one-loop corrections as shown in Figs. 4(b), 4(c), and 4(d). When Higgs boson's mass becomes heavy, the results of the effective field theory method do not converge with the exact one-loop calculation. 
The reason for this anomaly might be related with our approximations we have used.

From these figures, generally speaking, we can draw the conclusion that the one-loop matching conditions with the one-loop RGE method is reliable to a certain degree, and the effective theory description is valid even Higgs boson is relatively light.

\section{DISCUSSIONS AND CONCLUSIONS}

In this article we study the renormalization of the nonlinear $S U(2)$ Higgs model in the $\overline{M S}$ renormalization scheme. In order to get the divergent structure given in Eq. (4.14), we prescribe an appropriate parameterization for the Goldstone bosons from the phase angles. We realize that the EOMs of both gauge and Higgs bosons are very crucial in order to verify that the $S U(2)$ Higgs model in its nonlinear form is renormalizable.

We provide the exact one-loop calculation of the anomalous couplings and hence give the matching conditions up to one-loop level. It is worth mentioning that these matching conditions are also valid for the relatively light Higgs sector.

Our analysis shows that at the one-loop order, the $m_{H}$ independent part which comes from the contribution of gauge and Goldstone bosons is equally important, and bears the same order of the magnitude to the part that is dependent on the Higgs boson's mass. We observe that even for the region where the Higgs boson is relatively light, the effective description is still reliable to a certain degree.

Our numerical analysis shows that the predictions of the matching conditions at the one-loop level are better than those achieved at the tree-level. By comparing the predictions of the one-loop RGE and one-loop exact computation, we find that even for a relatively light Higgs boson, operators up to $O\left(p^{4}\right)$ in the gauge boson sector can accounts for the features of the Higgs boson in terms of the effective anomalous couplings to a certain degree.

To compare our results for the anomalous couplings, we have to seek a proper correspondence between the coordinate space and momentum space calculations. Also we have to remember that the existing calculations in the literature [2] accounts only for the $m_{H}$ dependent parts for the very heavy Higgs boson region. Our results agree with those given in [2] in the decoupling limit if the proper correspondence is made between the $\overline{M S}$ renormalization scheme in the coordinate space and the On-Shell renormalization scheme in the momentum space formalisms.

Before closing, we would like to address few subtleties and limitations of our calculation

1. Gauge operator of the Goldstone bosons: The gauge potential for the Goldstone boson can be defined as 


$$
\Gamma_{\mu}^{\xi}=-i \bar{W}_{\mu}^{a b}+\delta^{a b} \frac{\partial_{\mu} \bar{H}}{(v+\bar{H})} .
$$

It is interesting to note that the symmetric part does not contribute to the antisymmetric field strength tensor. However, this fact renders that the trace of the gauge potential defined in the gauge covariant differential operator is non-vanishing.

2. Usage of the EOM : In order to eliminate the linear terms of quantum fields in the Lagrangian, the EOM of background fields were used. We mentioned earlier, the feature of such application of the EOM (4.5) to evaluate the finite contributions of the term $\sigma_{\xi}^{a b}$ in the definition of the Goldstone boson's propagator in Eq. (4.11b). However, we have restricted ourselves from using the EOM of the Higgs boson elsewhere. For example, the EOM of the Higgs boson is not used in the term $\vec{X} \square_{W}^{-1} \bar{X} \square_{\xi}^{-1}$ in the effective irreducible vertex functional in Eq. (4.12), which contains the term $\partial_{\alpha} \bar{H} \partial^{\alpha} \partial^{2} \bar{H}$. This would imply the dropping of the higher order terms of the momentum expansion of the scalar loop integral $B_{0}\left(p^{2}, m_{1}^{2}, m_{2}^{2}\right)$. And this is consistent within the framework of coordinate space calculations. The use of the EOM of Higgs does not change the physical amplitudes of the theory as has been demonstrated in reference $[19,20]$.

3. Higher order contributions : In our computation procedure, we find that higher order operator affects the calculation of the finite contribution of the lower order operator $\operatorname{tr}\left[V_{\mu} V^{\mu}\right] \operatorname{tr}\left[V_{\nu} V^{\nu}\right]$. For instance, one of the operator of the order of $O\left(p^{5}\right)$ $\partial_{\alpha} \partial_{\beta} \bar{H} \bar{W}^{\alpha} \bar{W}^{\beta}$ can be expressed as

$$
\begin{aligned}
\int \partial_{\alpha} \partial_{\beta} \bar{H} \bar{W}^{\alpha} \bar{W}^{\beta}= & \int \frac{1}{2} \partial^{2} \overline{H W} \cdot \bar{W}-\int \frac{g^{2}}{4} \overline{H W}^{\beta}\left(\partial^{2} g_{\alpha \beta}-\partial_{\beta} \partial_{\alpha}\right) \bar{W}^{\alpha} \\
& +\int \overline{H W}_{\mu \nu}^{a} \bar{W}^{\mu \nu, a}+\cdots
\end{aligned}
$$

and by using the EOMs of Higgs and gauge bosons in the above equation, the first term contribute to $\operatorname{tr}\left[V_{\mu} V^{\mu}\right] \operatorname{tr}\left[V_{\nu} V^{\nu}\right]$. From this example, we realize that the information on the form of the complete set of higher order operators (say $O\left(p^{5}\right)$, $O\left(p^{6}\right)$, and so on) is necessary in order to determine the contributions to the lower order operators, and this fact indeed complicates our computation procedure. This kind of conributions will affect the value of $d_{3}$. However, due to this complication, we omit such contributions from higher order operators.

4. Matching in the coordinate space: The matching conditions are constructed to establish the connection between the parameters of the underlying full theory and the 
effective theory. The consequence of the matching of these two theories directly, would render the couplings of the effective theory non-local. In order to extract a local interaction theory, it is customary to expand the non-local effective couplings as so to get a local interaction theory truncated to a specified order. This is, a common practice for the three and four points functions. For two point functions, the correspondence is not simple. In order to realize the On-Shell renormalization scheme in the coordinate space, we have to sum over all the higher order terms which contribute to the two point functions, which then proves to be a disaster for our computation. However, for the two point functions, by using the correspondence between the coordinate space and the momentum space as given in appendix D, we can successfully collect all the relevant terms. This makes the On-Shell renormalization scheme workable in the coordinate space.

Next we would like to address the more realistic $S U(2) \times U(1)$ model in our project of trying to understand the full potential of the effective electro-weak Lagrangian.

\section{ACKNOWLEDGMENTS}

S. Dutta would like to thank the Science and Engineering Research Council, Department of Science and Technology, Government of India for the partial financial support. The work of K. Hagiwara and Q.S. Yan are supported in part by Grant-in-Aid Scientific Research from Ministry of Education, Culture, Science and Technology of Japan. The work of Q.S. Yan is partially supported by the Japan Society for the Promotion of Science fellowship program.

\section{APPENDIX A: SHORT DISTANCE EXPANSION APPROXIMATION}

In heat kernel method, the propagators for vector, Goldstone and Higgs bosons are defined as

$$
\begin{aligned}
\left\langle x\left|\square_{W ; \mu \nu}^{-1, a b}\right| y\right\rangle & =\int_{0}^{\infty} \frac{d \tau}{(4 \pi \tau)^{\frac{d}{2}}} \exp \left(-m_{W}^{2} \tau\right) \exp \left(-\frac{z^{2}}{4 \tau}\right) H_{W}^{\mu \nu, a b}(x, y ; \tau) \\
\left\langle x\left|\square_{\xi}^{-1, a b}\right| y\right\rangle & =\int_{0}^{\infty} \frac{d \tau}{(4 \pi \tau)^{\frac{d}{2}}} \exp \left(-m_{W}^{2} \tau\right) \exp \left(-\frac{z^{2}}{4 \tau}\right) H_{\xi}^{a b}(x, y ; \tau) \\
\left\langle x\left|\square_{H}^{-1}\right| y\right\rangle & =\int_{0}^{\infty} \frac{d \tau}{(4 \pi \tau)^{\frac{d}{2}}} \exp \left(-m_{H}^{2} \tau\right) \exp \left(-\frac{z^{2}}{4 \tau}\right) H_{H}(x, y ; \tau)
\end{aligned}
$$


where $z=y-x$ is short distance variable. The integral over the proper time $\tau$ and the factor $\exp \left[-z^{2} /(4 \tau)\right] /(4 \pi \tau)^{\frac{d}{2}}$ contains the quadratic divergent part of the propagator. The analytic behaviour of the function $H_{i}(x, y ; \tau)$ ( where $i \equiv W, \xi, h$ ) with reference to the variables $z$ and $\tau$, enables its expansion in terms of these variables.

The expansion of $H_{i}(x, y ; \tau)$ in $\tau$ is given as

$$
H_{i}(x, y ; \tau)=H_{i, 0}(x, y)+H_{i, 1}(x, y) \tau+H_{i, 2}(x, y) \tau^{2}+\cdots,
$$

where $H_{i, 0}(x, y), H_{i, 1}(x, y)$, and, $H_{i, 2}(x, y)$ are known to be Seeley-De Witt coefficients [21]. The coefficient $H_{0}(x, y)$ is the pure Wilson phase factor, which indicates the phase change of a quantum state when propagating from the point $y$ to the point $x$ and reads

$$
H_{i, 0}(x, y)=C_{i} \exp \left(-\int_{y}^{x} \Gamma_{i}(z) \cdot d z\right)
$$

where $\Gamma_{i}(z)$ is the affine connection ( dependent on the group representation of the quantum states ) defined on the coordinate point $z$ and $C_{i}$ is the Lorentz structure related with the spin state of the ' $i$ ' boson. For gauge bosons $C_{i} \equiv g^{\mu \nu}$, which in Euclidean space is essentially $\delta^{\mu \nu}$, and for Goldstone and Higgs bosons $C_{i}=1$. In the coincidence limit, we have $\left.H_{i, 0}(x, y)\right|_{y \rightarrow x}=1$.

The higher order Seeley-De Witt coefficients are determined by the recursion relation

$$
\left(1+n+z_{\mu} D_{i, x}^{\mu}\right) H_{i, n+1}(x, y)+\left(-D_{i, x}^{2}+\sigma_{i}\right) H_{i, n}(x, y)=0 .
$$

In the coincidence limit, the second and third Seeley-De Witt coefficients determined by the recursion relation given in Eq. (A.4) are expressed as

$$
\begin{aligned}
& H_{i, 1}(x)=-\sigma_{i}, \\
& H_{i, 2}(x)=\frac{1}{12} \Gamma_{i, \mu \nu} \Gamma_{i}^{\mu \nu} .
\end{aligned}
$$

The short distance expansion of $H_{i}(x, y ; \tau)$ around the coordinate $x$ is the ordinary Taylor expansion, which can be expressed as

$$
H_{i}(x, y)=\left.H_{i}(x, y)\right|_{x=y}+\left.z^{\alpha} \partial_{\alpha} H_{i}(x, y)\right|_{x=y}+\left.\frac{1}{2} z^{\alpha} z^{\beta} \partial_{\alpha} \partial_{\beta} H_{i}(x, y)\right|_{x=y}+\cdots .
$$

We make use of this expansion in our calculation.

We also define the operation of the covariant differential operator $D_{i, x}^{\alpha}$ on its corresponding propagator defined by $-D_{i}^{2}+m_{i}^{2}+\sigma_{i}$ in terms of the integral

$$
\begin{aligned}
\left\langle x\left|D_{i, x}^{\alpha} \square_{i}^{-1}\right| y\right\rangle= & \int_{x^{\prime}}\left\langle x\left|D_{i, x}^{\alpha}\right| x^{\prime}\right\rangle\left\langle x^{\prime}\left|\square_{i}^{-1}\right| y\right\rangle \\
= & \int d \lambda \frac{1}{(4 \pi \lambda)^{d / 2}} \exp \left\{-m_{i}^{2} \lambda\right\} \exp \left(-\frac{z^{2}}{4 \lambda}\right) \\
& \times\left(\frac{z^{\alpha}}{2 \lambda}+D_{i}^{\alpha}\right) H_{i}(x, y ; \lambda) .
\end{aligned}
$$


Using this definition we can show

$$
\begin{aligned}
\left.D_{i, x}^{\alpha} H_{i, 0}(x, y)\right|_{y \rightarrow x} & =0, \\
\left.D_{i, x}^{\alpha} D_{i, x}^{\beta} H_{i, 0}(x, y)\right|_{y \rightarrow x} & =\frac{1}{2} \Gamma_{i, \alpha \beta} .
\end{aligned}
$$

The short distance covariant expansion of operators $X(x)$ given in Eqs. (4.10f-4.10n) are defined as operator $I(x, y)$ which can be expressed as

$$
\begin{aligned}
I(x, y) & =H_{i, 0}(x, y) X(y) H_{j, 0}(y, x) \\
& =X(x)+z^{\alpha} D_{\alpha} X(x)+\frac{z^{\alpha} z^{\beta}}{2} D_{\alpha} D_{\beta} X(x)+\cdots .
\end{aligned}
$$

Here $H_{i(j), 0}$ means the first Seeley-De Witt coefficient of the particle $i(j)$, and

$$
\begin{aligned}
D_{\alpha} X= & \partial_{\alpha} X+\Gamma_{i, \alpha} X-X \Gamma_{j, \alpha} \\
D_{\alpha} D_{\beta} X= & \partial_{\alpha} \partial_{\beta} X+\Gamma_{i, \alpha} \Gamma_{i, \beta} X+X \Gamma_{j, \alpha} \Gamma_{j, \beta}-2 \Gamma_{i, \alpha} X \Gamma_{j, \beta} \\
& +2 \Gamma_{i, \alpha} \partial_{\beta} X-2 \partial_{\alpha} X \Gamma_{j, \beta}+\partial_{\alpha} \Gamma_{i, \beta} X-X \partial_{\alpha} \Gamma_{j, \beta} .
\end{aligned}
$$

\section{APPENDIX B: DIVERGENT AND FINITE TERMS OF THE NONLINEAR SU(2) HIGGS MODEL}

In order to extract all the relevant terms up to $O\left(p^{4}\right)$ (both divergent and finite), we need to introduce an auxiliary dimension counting rule in coordinate space calculation, which reads as

$$
\left[\bar{W}_{\mu}^{s}\right]_{a}=\left[\partial_{\mu}\right]_{a}=\left[D_{\mu}\right]_{a}=[\bar{H}]_{a}=1,[v]_{a}=0
$$

The divergence counting rule of the integral over the coordinate space $z$ and the proper time $\tau$ can be established as

$$
\left[z^{\mu}\right]_{d}=1,[\tau]_{d}=2
$$

The relevant terms from the trace of single propagator $\ln \square_{i}$ given in Eq. (4.12)) are calculated by standard procedure and given as

$$
\begin{aligned}
\ln \square_{W}= & -\frac{\left(\mu^{2}\right)^{(\epsilon / 2)}}{16 \pi^{2}} \int_{x}\left\{d C_{a d}\left(m_{W}^{2}\right)^{\frac{d}{2}} \Gamma\left(-\frac{d}{2}\right)-\left(m_{W}^{2}\right)^{\frac{d}{2}-1} \Gamma\left(1-\frac{d}{2}\right) \sigma_{W}^{\mu \mu, a a}\right. \\
& \left.+\left(m_{W}^{2}\right)^{\frac{d}{2}-2} \Gamma\left(2-\frac{d}{2}\right)\left[\frac{d C_{a d}}{12} \Gamma_{\mu \nu} \Gamma^{\mu \nu}+\frac{1}{2} \sigma_{W}^{\mu \nu, a b} \sigma_{W}^{\nu \mu, b a}\right]+\cdots\right\},
\end{aligned}
$$




$$
\begin{aligned}
\ln \square_{\xi}= & -\frac{\left(\mu^{2}\right)^{(\epsilon / 2)}}{16 \pi^{2}} \int_{x}\left\{C_{a d}\left(m_{W}^{2}\right)^{\frac{d}{2}} \Gamma\left(-\frac{d}{2}\right)-\left(m_{W}^{2}\right)^{\frac{d}{2}-1} \Gamma\left(1-\frac{d}{2}\right) \sigma_{\xi}^{a a}\right. \\
& \left.+\left(m_{W}^{2}\right)^{\frac{d}{2}-2} \Gamma\left(2-\frac{d}{2}\right)\left[\frac{C_{a d}}{12} \Gamma_{\xi, \mu \nu} \Gamma_{\xi}^{\mu \nu}+\frac{1}{2} \sigma_{\xi}^{a b} \sigma_{\xi}^{b a}\right]+\cdots\right\}, \\
\ln \square_{H}= & -\frac{\left(\mu^{2}\right)^{(\epsilon / 2)}}{16 \pi^{2}} \int_{x}\left\{\left(m_{H}^{2}\right)^{\frac{d}{2}} \Gamma\left(-\frac{d}{2}\right)-\left(m_{H}^{2}\right)^{\frac{d}{2}-1} \Gamma\left(1-\frac{d}{2}\right) \sigma_{H}\right. \\
& \left.+\left(m_{H}^{2}\right)^{\frac{d}{2}-2} \Gamma\left(2-\frac{d}{2}\right)\left[\frac{1}{2} \sigma_{H}^{2}\right]+\cdots\right\}, \\
\ln \square_{c}= & -\frac{\left(\mu^{2}\right)^{(\epsilon / 2)}}{16 \pi^{2}} \int_{x}\left\{C_{a d}\left(m_{W}^{2}\right)^{\frac{d}{2}} \Gamma\left(-\frac{d}{2}\right)-\left(m_{W}^{2}\right)^{\frac{d}{2}-1} \Gamma\left(1-\frac{d}{2}\right) \sigma_{c c}^{a a}\right. \\
& \left.+\left(m_{W}^{2}\right)^{\frac{d}{2}-2} \Gamma\left(2-\frac{d}{2}\right)\left[\frac{C_{a d}}{12} \Gamma_{\mu \nu} \Gamma^{\mu \nu}+\frac{1}{2} \sigma_{c c}^{a b} \sigma_{c c}^{b a}\right]+\cdots\right\},
\end{aligned}
$$

where $C_{a d}=\sum_{i} \operatorname{tr}\left(t_{a d}^{i} t_{a d}^{i}\right)$, and $t_{a d}^{i}$ are the matrices of the Lie algebra in the adjoint representation.

Calculations of the traces with more than one propagator are tedious but straightforward. However, here we work out the calculation of one trace involving two propagators. For example, consider the term $\operatorname{Tr}\left(\vec{X} \square_{W}^{-1} \overleftarrow{X} \square_{H}^{-1}\right)$.

$$
\begin{aligned}
S_{W H}= & \operatorname{Tr}\left(\vec{X}_{H} \square_{W}^{-1} \overleftarrow{X}_{H} \square_{H}^{-1}\right) \\
= & \int_{x} \operatorname{tr}\left(\left\langle x\left|\vec{X}_{H} \square_{W}^{-1} \overleftarrow{X}_{H} \square_{H}^{-1}\right| x\right\rangle\right) \\
= & \int_{x} \int_{y} \operatorname{tr}\left(\vec{X}_{H}(x)\left\langle x\left|\square_{W}^{-1}\right| y\right\rangle \overleftarrow{X}_{H}(y)\left\langle y\left|\square_{H}^{-1}\right| x\right\rangle\right) \\
= & \int_{x} \int_{z} \int_{\lambda_{1}} \int_{\lambda_{2}} \exp \left\{-m_{W}^{2} \lambda_{1}-m_{H}^{2} \lambda_{2}\right\} \exp \left\{-z^{2}\left(\frac{\lambda_{1}+\lambda_{2}}{4 \lambda_{1} \lambda_{2}}\right)\right\} \\
& \times \vec{X}_{H}(x)^{\mu, a} H_{W}^{\mu \nu, a b}\left(x, y, \lambda_{1}\right) \overleftarrow{X}_{H}(y)^{\nu, a} H_{H}\left(y, x ; \lambda_{2}\right) .
\end{aligned}
$$

Using the short distance expansion given in Eq. (A.9) and the auxiliary dimension counting rule given in Eq. (B.1), we can easily find that the relevant terms in $S_{W H}$ can be expressed as

$$
\begin{aligned}
S_{W H}= & \int_{x} \int_{z} \int_{\lambda_{1}} \int_{\lambda_{2}} \exp \left\{-m_{W}^{2} \lambda_{1}-m_{H}^{2} \lambda_{2}\right\} \exp \left\{-z^{2}\left(\frac{\lambda_{1}+\lambda_{2}}{4 \lambda_{1} \lambda_{2}}\right)\right\} \\
& \times\left\{\vec{X}_{H}(x)^{\mu, a} \overleftarrow{X}_{H}(x)^{\mu, a}+\lambda_{1} \vec{X}_{H}(x)^{\mu, a} H_{W, 1}^{\mu \nu, a b}(x) \overleftarrow{X}_{H}(x)^{\nu, b}\right. \\
& \left.+\lambda_{2} \vec{X}_{H}(x)^{\mu, a} \overleftarrow{X}_{H}(x)^{\mu, a} H_{H, 1}(x)+\frac{z^{\alpha} z^{\beta}}{2} \vec{X}_{H}(x)^{\mu, a} D_{\alpha}^{a b} D_{\beta}^{b c} \overleftarrow{X}_{H}(x)^{\nu, c}+\left(O\left(p^{5}\right)\right)\right\} .
\end{aligned}
$$

After performing the integral over $z$ and proper times $\lambda_{1}$ and $\lambda_{2}$, we arrive at 


$$
\begin{aligned}
S_{W H}= & l_{f} \int_{x}\left\{\vec{X}_{H}(x)^{\mu, a} \overleftarrow{X}_{H}(x)^{\mu, a} B_{0, W H}+\vec{X}_{H}(x)^{\mu, a} H_{W, 1}^{\mu \nu, a b}(x) \overleftarrow{X}_{H}(x)^{\nu, b} C_{1, W H}\right. \\
& \left.+\vec{X}_{H}(x)^{\mu, a} \overleftarrow{X}_{H}(x)^{\mu, a} H_{H, 1}^{c a}(x) C_{2, W H}+\vec{X}_{H}(x)^{\mu, a} D_{\alpha}^{a b} D_{\alpha}^{b c} \overleftarrow{X}_{H}(x)^{\nu, c} C_{3, W H}+\cdots\right\},
\end{aligned}
$$

where $l_{f}$ is the one-loop factor $1 /\left(16 \pi^{2}\right)$.

For all other traces given in Eq. (4.12) we list the terms contributing up to the order of $O\left(p^{4}\right)$

$$
\begin{aligned}
& S_{W \xi}=\operatorname{Tr}\left(\vec{X}_{\xi} \square_{W}^{-1} \overleftarrow{X}_{\xi} \square_{\xi}^{-1}\right) \\
& =l_{f} \int_{x}\left\{\vec{X}_{\xi}(x)^{\mu, a b} \overleftarrow{X}_{\xi}(x)^{\mu, b a} B_{0, W \xi}+\vec{X}_{\xi}(x)^{\mu, a b} H_{W, 1}^{\mu \nu, b c}(x) \overleftarrow{X}_{\xi}(x)^{\nu, c a} C_{1, W \xi}\right. \\
& +\vec{X}_{\xi}(x)^{\mu, a b} \overleftarrow{X}_{\xi}(x)^{\mu, b c} H_{\xi, 1}^{c a}(x) C_{2, W \xi} \\
& \left.+\vec{X}_{\xi}(x)^{\mu, a b} D_{\alpha}^{b c} D_{\alpha}^{c d} \overleftarrow{X}_{\xi}(x)^{n u, d a} C_{3, W \xi}+\cdots\right\} \text {, } \\
& S_{\xi H}=\operatorname{Tr}\left(\vec{X}_{\xi} \square_{\xi}^{-1} \overleftarrow{X}_{\xi} \square_{H}^{-1}\right) \\
& =l_{f} \int_{x}\left\{\frac{1}{2} \vec{X}(x)^{\alpha, a} \overleftarrow{X}(x)^{\alpha, a} A_{0, \xi H}+\frac{1}{2} \vec{X}(x)^{\alpha, a} H_{\xi, 1}^{a b}(x) \overleftarrow{X}(x)^{\alpha, b} B_{0, \xi H}\right. \\
& +\frac{1}{2} \vec{X}(x)^{\alpha, a} \overleftarrow{X}(x)^{\alpha, a} H_{H, 1}(x) B_{1, \xi H}+\frac{g^{\alpha \beta \alpha^{\prime} \beta^{\prime}}}{2} \vec{X}(x)^{\alpha, a} D_{\alpha^{\prime}}^{a b} D_{\beta^{\prime}}^{b c} \overleftarrow{X}(x)^{\beta, c} B_{2, \xi H} \\
& \left.+\frac{1}{2} \vec{X}(x)^{\alpha, a} \Gamma_{\xi, \alpha \beta}^{a b} \overleftarrow{X}(x)^{\beta, b} B_{3, \xi H}+\cdots\right\} \\
& S_{W \xi H}=\operatorname{Tr}\left(\vec{X}_{H} \square_{W}^{-1} \overleftarrow{X}_{\xi} \square_{\xi}^{-1} \bar{X}_{H}^{-1}\right) \\
& =l_{f} \int_{x}\left\{\vec{X}_{H}(x)^{\mu, a} D_{\alpha}^{a b} \overleftarrow{X}_{x i}(x)^{\mu, b c} \vec{X}(x)^{\alpha, c} C_{0, W \xi H}\right. \\
& +\vec{X}_{H}(x)^{\mu, a} \stackrel{\leftarrow}{X}_{\xi}^{\mu, a b}(x) D_{\alpha}^{b c} \overleftarrow{X}(x)^{\alpha, c} C_{1, W \xi H} \\
& \left.+\vec{X}_{H}(x)^{\mu, a} \overleftarrow{X}_{\xi}(x)^{\mu, a} \vec{X}_{0} C_{2, W \xi H}+\cdots\right\} \text {, } \\
& S_{\xi W H}=\operatorname{Tr}\left(\vec{X} \square_{\xi}^{-1} \overleftarrow{X}_{\xi} \square_{W}^{-1} \overleftarrow{X}_{H} \square_{H}^{-1}\right) \\
& =l_{f} \int_{x}\left\{\vec{X}(x)^{\alpha, a} D_{\alpha}^{a b} \overleftarrow{X}_{\xi}(x)^{\mu, b c} \overleftarrow{X}_{H}(x)^{\mu, c} C_{0, \xi W H}\right. \\
& +\vec{X}(x)^{\alpha, a} \overleftarrow{X}_{\xi}(x)^{\mu, a b} D_{\alpha}^{b c} \overleftarrow{X}_{H}(x)^{\alpha, c} C_{1, \xi W H} \\
& \left.+\vec{X}(x)_{0} \overleftarrow{X}_{\xi}(x)^{\mu, a} \overleftarrow{X}_{H}(x)^{\mu, a} C_{2, \xi W H}+\cdots\right\}, \\
& S_{W \xi W \xi}=\operatorname{Tr}\left(\vec{X}_{\xi} \square_{W}^{-1} \overleftarrow{X}_{\xi} \square_{\xi}^{-1} \vec{X}_{\xi} \square_{W}^{-1} \overleftarrow{X}_{\xi} \square_{\xi}^{-1}\right) \\
& =l_{f} \int_{x}\left\{\vec{X}_{\xi}(x)^{\mu, a b} \overleftarrow{X}_{\xi}(x)^{\mu, b c} \vec{X}_{\xi}(x)^{\nu, c d} \overleftarrow{X}_{\xi}(x)^{\nu, d a} D_{0, W \xi W \xi}+\cdots\right\} \text {, } \\
& S_{W H W H}=\operatorname{Tr}\left(\vec{X}_{H} \square_{W}^{-1} \overleftarrow{X}_{H} \square_{H}^{-1} \vec{X}_{H} \square_{W}^{-1} \overleftarrow{X}_{H} \square_{H}^{-1}\right) \\
& =l_{f} \int_{x}\left\{\vec{X}_{H}(x)^{\mu, a} \overleftarrow{X}_{H}(x)^{\mu, a} \vec{X}_{H}(x)^{\nu, b} \overleftarrow{X}_{H}(x)^{\nu, b} D_{0, W H W H}+\cdots\right\} \text {, }
\end{aligned}
$$




$$
\begin{aligned}
& S_{\xi H \xi H}=\operatorname{Tr}\left(\vec{X} \square_{\xi}^{-1} \overleftarrow{X} \square_{H}^{-1} \vec{X} \square_{\xi}^{-1} \overleftarrow{X} \square_{H}^{-1}\right) \\
& =l_{f} \int_{x}\left\{\frac{g^{\alpha \beta \alpha^{\prime} \beta^{\prime}}}{4} \overleftarrow{X}(x)^{\alpha, a} \vec{X}(x)^{\beta, a} \overleftarrow{X}(x)^{\alpha^{\prime}, b} \vec{X}(x)^{\beta^{\prime}, b} B_{0, \xi H \xi H}+\cdots\right\}, \\
& S_{W \xi W H}=\operatorname{Tr}\left(\vec{X}_{H} \square_{W}^{-1} \overleftarrow{X}_{\xi} \square_{\xi}^{-1} \vec{X}_{\xi} \square_{W}^{-1} \overleftarrow{X}_{H} \square_{H}^{-1}\right) \\
& =l_{f} \int_{x}\left\{\vec{X}_{H}(x)^{\mu, a b} \overleftarrow{X}_{\xi}(x)^{\mu, b c} \vec{X}_{\xi}(x)^{\nu, c d} \overleftarrow{X}_{H}(x)^{\nu, d} D_{0, W \xi W H}+\cdots\right\} \text {, } \\
& S_{\xi W \xi H}=\operatorname{Tr}\left(\vec{X} \square_{\xi}^{-1} \overleftarrow{X}_{\xi} \square_{W}^{-1} \vec{X}_{\xi} \square_{\xi}^{-1} \overleftarrow{X}_{H}^{-1}\right) \\
& =l_{f} \int_{x}\left\{\vec{X}(x)^{\alpha, a} \overleftarrow{X}_{\xi}(x)^{\mu, a b} \vec{X}_{\xi}(x)^{\mu, b c} \vec{X}(x)^{\alpha, c} C_{0, \xi W \xi H}+\cdots\right\} \text {, } \\
& S_{\xi H W H}=\operatorname{Tr}\left(\vec{X} \square_{\xi}^{-1} \overleftarrow{X}_{H}^{-1} \vec{X}_{H} \square_{W}^{-1} \overleftarrow{X}_{H} \square_{H}^{-1}\right) \\
& =l_{f} \int_{x}\left\{\vec{X}(x)^{\alpha, a} \overleftarrow{X}(x)^{\alpha, a} \vec{X}_{H}(x)^{\mu, b} \overleftarrow{X}_{H}(x)^{\mu, b} C_{0, \xi H W H}+\cdots\right\} \text {. }
\end{aligned}
$$

The scalar integrals in Eqs. (B.7a-B.7j) are given as

$$
\begin{aligned}
B_{0, W H} & =\frac{2}{\bar{\epsilon}}+\ln \left(\frac{\mu^{2}}{m_{W}^{2}}\right)+1-\frac{r}{r-1} \ln (r), \\
C_{1, W H} & =\frac{1}{m_{W}^{2}}\left\{-\frac{1}{r-1}+\frac{r}{(r-1)^{2}} \ln (r)\right\}, \\
C_{2, W H} & =\frac{1}{m_{W}^{2}}\left\{\frac{1}{r-1}-\frac{1}{(r-1)^{2}} \ln (r)\right\} \\
C_{3, W H} & =\frac{1}{m_{W}^{2}}\left\{\frac{r+1}{2(r-1)^{2}}-\frac{r}{(r-1)^{3}} \ln (r)\right\}, \\
B_{0, W \xi} & =\frac{2}{\bar{\epsilon}}+\ln \left(\frac{\mu^{2}}{m_{W}^{2}}\right), \\
C_{1, W \xi} & =\frac{1}{2 m_{W}^{2}}, \\
C_{2, W \xi} & =\frac{1}{2 m_{W}^{2}}, \\
C_{3, W \xi} & =\frac{1}{6 m_{W}^{2}}, \\
A_{0, \xi H} & =m_{W}^{2}\left\{\frac{(1+r)}{2}\left(\frac{2}{\bar{\epsilon}}+\ln \left(\frac{\mu^{2}}{m_{W}^{2}}\right)\right)+\frac{3(r+1)}{4}-\frac{r^{2}}{2(r-1)} \ln (r)\right\}, \\
B_{0, \xi H} & =-\frac{1}{2} \frac{2}{\bar{\epsilon}}-\frac{1}{2} \ln \left(\frac{\mu^{2}}{m_{W}^{2}}\right)+\frac{1-3 r}{4(r-1)}+\frac{r^{2}}{2(r-1)^{2}} \ln (r), \\
B_{2, \xi H} & =-\frac{1}{6} \frac{2}{\bar{\epsilon}}-\frac{1}{6} \ln \left(\frac{\mu^{2}}{m_{W}^{2}}\right)+\frac{-5 r^{2}+22 r-5}{36(r-1)^{2}}+\frac{(r-3) r^{2}}{6(r-1)^{3}} \ln (r), \\
B_{1, \xi H} & =-\frac{2}{2} \frac{1}{\bar{\epsilon}}-\frac{1}{2} \ln \left(\frac{\mu^{2}}{m_{W}^{2}}\right)+\frac{3-2)}{4(r-1)} \ln (r), \\
&
\end{aligned}
$$




$$
\begin{aligned}
B_{3, \xi H} & =B_{0, \xi H} \\
C_{0, W \xi H} & =\frac{1}{m_{W}^{2}}\left(\frac{3 r-1}{4(r-1)^{2}}-\frac{r^{2}}{2(r-1)^{3}} \ln (r)\right), \\
C_{1, W \xi H} & =\frac{1}{m_{W}^{2}}\left(\frac{1-3 r}{4(r-1)^{2}}+\frac{r^{2}}{2(r-1)^{3}} \ln (r)\right), \\
C_{2, W \xi H} & =\frac{1}{m_{W}^{2}}\left(-\frac{1}{r-1}+\frac{r}{(r-1)^{2}} \ln (r)\right) \\
C_{0, \xi W H} & =\frac{1}{m_{W}^{2}}\left(-\frac{r-3}{4(r-1)^{2}}+\frac{r(r-2)}{2(r-1)^{3}} \ln (r)\right), \\
C_{1, \xi W H} & =\frac{1}{m_{W}^{2}}\left(\frac{3 r-1}{4(r-1)^{2}}-\frac{r^{2}}{2(r-1)^{3}} \ln (r)\right), \\
C_{2, \xi W H} & =C_{2, W \xi H}, \\
D_{0, W \xi W \xi} & =\frac{1}{m_{W}^{4}} \frac{1}{6}, \\
D_{0, W H W H} & =\frac{1}{m_{W}^{4}}\left(-\frac{2}{(r-1)^{2}}+\frac{r+1}{(r-1)^{3}} \ln (r)\right), \\
C_{0, \xi H W H} & =\frac{1}{m_{W}^{2}}\left(\frac{r+1}{2(r-1)^{2}}-\frac{r}{(r-1)^{3}} \ln (r)\right), \\
B_{0, \xi H \xi H} & =\frac{1}{6} \frac{2}{\bar{\epsilon}}+\frac{1}{6} \ln \left(\frac{\mu^{2}}{m_{W}^{2}}\right)-\frac{r r^{2}+22 r-5}{36(r-1)^{2}}-\frac{r^{2}(r-3)}{6(r-1)^{3}} \ln (r), \\
D_{0, W \xi W H} & =\frac{1}{m_{W}^{4}}\left(\frac{r+1}{2(r-1)^{2}}-\frac{r}{(r-1)^{3}} \ln (r)\right), \\
m_{W}^{2} & \left.\frac{1}{4(r-1)^{2}}+\frac{r^{2}}{2(r-1)^{3}} \ln (r)\right), \\
& =1 r
\end{aligned}
$$

here $A, B, C$ and $D$ are the scalar integrals.

\section{APPENDIX C: DIVERGENCES OF THE ONE-LOOP SU(2) CHIRAL LAGRANGIAN}

In reference [9] authors have provided the details. We found couple of errors in this calculation and here we rectify them. Following the BFM we split the vector and Goldstone fields of the chiral Lagrangian as given in Eq. (3.1a) into classical and quantum parts. We collect the mixing operators between quantum vector bosons and Goldstone bosons into the standard form, and then we can get the one-loop irreducible generating functional which is expressed as

$$
\Gamma_{1-\text { loop }}^{c h l}=-\frac{1}{2} \quad\left[\operatorname{Tr} \ln \square_{W}+\operatorname{Tr} \ln \square_{\xi}-2 \operatorname{Tr} \ln \square_{c}\right.
$$




$$
\begin{aligned}
& +\operatorname{Tr}\left(X^{\alpha \beta} d_{\alpha} d_{\beta} \square_{\xi}^{-1}\right)-\frac{1}{2} \operatorname{Tr}\left(X^{\alpha \beta} d_{\alpha} d_{\beta} \square_{\xi}^{-1} X^{\alpha^{\prime} \beta^{\prime}} d_{\alpha^{\prime}} d_{\beta^{\prime}} \square_{\xi}^{-1}\right) \\
& -\operatorname{Tr}\left(\vec{X}_{\xi} \square_{W}^{-1} \overleftarrow{X}_{\xi} \square_{\xi}^{-1}\right)+\operatorname{Tr}\left(\vec{X}_{\xi} \square_{W}^{-1} \overleftarrow{X}_{\xi} \square_{\xi}^{-1} X^{\alpha \beta} d_{\alpha} d_{\beta} \square_{\xi}^{-1}\right) \\
& \left.-\frac{1}{2} \operatorname{Tr}\left(\vec{X}_{\xi} \square_{W}^{-1} \overleftarrow{X}_{\xi} \square_{\xi}^{-1} \vec{X}_{\xi} \square_{W}^{-1} \overleftarrow{X}_{\xi} \square_{\xi}^{-1}\right)+\cdots\right] .
\end{aligned}
$$

While operators in Eq. (C.1) are defined as

$$
\begin{aligned}
\square_{W W}^{\mu \nu, a b} & =\left(-D^{\prime 2, a b}+m_{W}^{2} \delta^{a b}\right) g^{\mu \nu}+\sigma_{W W}^{\mu \nu, a b}, \\
\square_{\xi \xi}^{\prime a b} & =\square_{\xi \xi}^{a b}+X^{\alpha, a c} d_{\alpha}^{c b}+X^{\alpha \beta, a c} d_{\alpha}^{c d} d_{\beta}^{d b}, \\
\square_{\xi \xi}^{a b} & =\left(-d^{2, a b}+\delta^{a b} m_{W}^{2}\right)+\sigma_{2, \xi \xi}^{a b}+\sigma_{4, \xi \xi}^{a b}, \\
\square_{\bar{c} c}^{a b} & =\left(-D^{\prime 2, a b}+m_{W}^{2} \delta^{a b}\right), \\
\overleftarrow{X}^{\mu, a b} & =\overleftarrow{X}_{\alpha \beta}^{\mu, a c} d^{\alpha, c d} d^{\beta, d b}+\overleftarrow{\complement}^{\mu \alpha, a c} d_{\alpha}^{c b}+\overleftarrow{X}_{01}^{\mu, a b}+\overleftarrow{X}_{03 Z}^{\mu, a b}+\partial_{\alpha} \overleftarrow{X}_{03 Y}^{\mu \alpha, a b}, \\
\vec{X}^{\nu, a b} & =\vec{X}_{\alpha \beta}^{\nu, a c} D^{\prime \alpha, c d} D^{\prime \beta, d b}+\vec{X}^{\nu \alpha, a c} D_{\alpha}^{\prime c b}+\vec{X}_{01}^{\nu, a b}+\vec{X}_{03 Z}^{\nu, a b}+\partial_{\alpha} \vec{X}_{03 Y}^{\nu \alpha, a b},
\end{aligned}
$$

the operators appearing in Eqs. (C.2a-C.2f) are defined in [9].

Since the trace log terms are similar to those given in Eqs. (B.3a-B.3d), below we list the divergent structures of the rest of terms in Eq. (C.1),

$$
\begin{aligned}
& S_{\xi, 1}=\operatorname{Tr}\left(X^{\alpha \beta} d_{\alpha} d_{\beta} \square_{\xi}^{-1}\right) \\
& =-\frac{1}{2} l_{f} \int_{x}\left\{g^{\alpha \beta} \operatorname{tr}\left(X^{\alpha \beta}\right) Q_{4}+g^{\alpha \beta} \operatorname{tr}\left(X^{\alpha \beta} H_{\xi, 1}\right) Q_{2}\right\}, \\
& S_{\xi, 2}=\operatorname{Tr}\left(X^{\alpha \beta} d_{\alpha} d_{\beta} \square_{\xi}^{-1} X^{\alpha^{\prime} \beta^{\prime}} d_{\alpha^{\prime}} d_{\beta^{\prime}} \square_{\xi}^{-1}\right) \\
& =l_{f} \int_{x}\left\{\frac{g^{\alpha \beta \alpha^{\prime} \beta^{\prime}}}{4} \operatorname{tr}\left(X^{\alpha \beta} X^{\alpha^{\prime} \beta^{\prime}}\right) Q_{4}\right\}, \\
& S_{v \xi \xi}=\operatorname{Tr}\left(\vec{X}_{\xi} \square_{W}^{-1} \overleftarrow{X}_{\xi} \square_{\xi}^{-1} X^{\alpha \beta} d_{\alpha} d_{\beta} \square_{\xi}^{-1}\right) \\
& =l_{f} \int_{x}\left\{-\frac{g^{\alpha \beta \alpha^{\prime} \beta^{\prime} \alpha^{\prime \prime} \beta^{\prime \prime}}}{8} \operatorname{tr}\left(\vec{X}^{\mu, \alpha \beta} \check{L}^{\mu, \alpha^{\prime} \beta^{\prime}} X^{\alpha^{\prime \prime} \beta^{\prime \prime}}\right) Q_{4}\right. \\
& \left.+\frac{g^{\alpha \beta \alpha^{\prime} \beta^{\prime}}}{4} \operatorname{tr}\left(\vec{X}^{\mu, \alpha \beta} \overleftarrow{\mathrm{X}}_{01}^{\mu} X^{\alpha^{\prime} \beta^{\prime}}+\vec{X}_{01}^{\mu} \overleftarrow{X}^{\mu, \alpha \beta} X^{\alpha^{\prime} \beta^{\prime}}\right) Q_{2}-\frac{g^{\alpha \beta}}{2} \operatorname{tr}\left(\vec{X}_{01}^{\mu} \overleftarrow{X}_{01}^{\mu} X^{\alpha \beta}\right) Q_{0}\right\} \\
& S_{v \xi v \xi}=\operatorname{Tr}\left(\vec{X}_{\xi} \square_{W}^{-1} \overleftarrow{X}_{\xi} \square_{\xi}^{-1} \vec{X}_{\xi} \square_{W}^{-1} \overleftarrow{X}_{\xi} \square_{\xi}^{-1}\right) \\
& =l_{f} \int_{x}\left\{\frac{g^{\alpha \beta \alpha^{\prime} \beta^{\prime} \alpha^{\prime \prime} \beta^{\prime \prime} \alpha^{\prime \prime \prime} \beta^{\prime \prime \prime}}}{16} \operatorname{tr}\left(\vec{X}^{\mu, \alpha \beta} \overleftarrow{X}^{\mu, \alpha^{\prime} \beta^{\prime}} \vec{X}^{\nu, \alpha^{\prime \prime} \beta^{\prime \prime}} \overleftarrow{X}^{\nu, \alpha^{\prime \prime \prime} \beta^{\prime \prime \prime \prime}}\right) Q_{4}\right.
\end{aligned}
$$

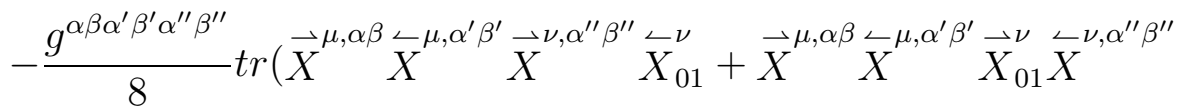

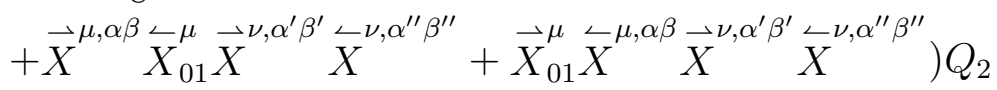




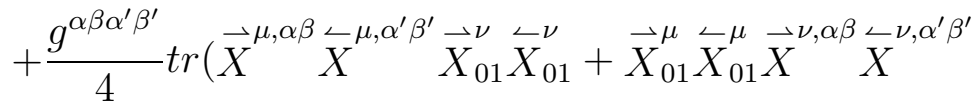

$$
\begin{aligned}
& +\vec{X}^{\mu, \alpha \beta} \stackrel{\leftarrow}{X}_{01}^{\mu} \vec{X}^{\nu, \alpha^{\prime} \beta^{\prime}} \stackrel{\breve{X}}{X}_{01}^{\nu}+\vec{X}_{01}^{\mu} \overleftarrow{X}^{\mu, \alpha \beta} \vec{X}_{01}^{\nu} \overleftarrow{X}^{\nu, \alpha^{\prime} \beta^{\prime}}
\end{aligned}
$$

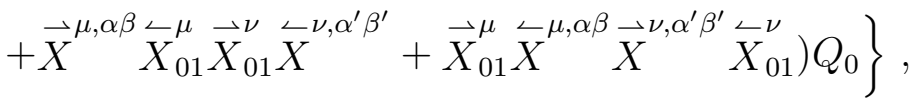

$$
\begin{aligned}
& S_{v \xi}=\operatorname{Tr}\left(\vec{X}_{\xi} \square_{W}^{-1} \overleftarrow{X}_{\xi} \square_{\xi}^{-1}\right) \\
& =l_{f} \int_{x}\left\{t_{A A}+t_{B B}+t_{C C}+t_{A B}+t_{A C}+t_{B C}\right\} \text {, } \\
& t_{A A}=\frac{1}{4} g^{\alpha \beta \alpha^{\prime} \beta^{\prime}} g^{\mu \nu} \operatorname{tr}\left[\vec{X}_{\alpha \beta}^{\mu} \overleftarrow{X}_{\alpha^{\prime} \beta^{\prime}}^{\nu}\right] Q_{4} \\
& +\frac{g^{\mu \nu}}{4}\left(\frac{g^{\alpha \beta \alpha^{\prime} \beta^{\prime} \delta \gamma}}{6}-\frac{g^{\alpha \beta} g^{\alpha^{\prime} \beta^{\prime} \delta \gamma}}{2}-\frac{g^{\alpha^{\prime} \beta^{\prime}} g^{\alpha \beta \delta \gamma}}{2}+g^{\alpha \beta} g^{\alpha^{\prime} \beta^{\prime}} g^{\delta \gamma}\right) \operatorname{tr}\left[\vec{X}_{\alpha \beta}^{\mu} D_{\delta} D_{\gamma} \overleftarrow{X}_{\alpha^{\prime} \beta^{\prime}}^{\nu}\right] Q_{2} \\
& +\frac{g^{\alpha \beta \alpha^{\prime} \beta^{\prime}}}{8}\left[g_{\mu \nu} \operatorname{tr}\left[\vec{X}_{\alpha \beta}^{\mu} \overleftarrow{X}_{\alpha^{\prime} \beta^{\prime}}^{\nu} H_{\xi, 1}\right]+g_{\mu \mu^{\prime}} g_{\nu \nu^{\prime}} \operatorname{tr}\left[\vec{X}_{\alpha \beta}^{\mu} H_{W, 1}^{\mu^{\prime} \nu^{\prime}} \overleftarrow{X}_{\alpha^{\prime} \beta^{\prime}}^{\nu}\right]\right] Q_{2}, \\
& t_{A B}=\frac{g_{\alpha^{\prime} \alpha^{\prime \prime}} g_{\mu \nu}}{2}\left(g^{\alpha \beta} g^{\alpha^{\prime} \beta^{\prime}}-\frac{1}{2} g^{\alpha \beta \alpha^{\prime} \beta^{\prime}}\right) \operatorname{tr}\left[\vec{X}_{\alpha \beta}^{\mu} D_{\beta^{\prime}} \breve{X}^{\nu \alpha^{\prime \prime}}-\vec{X}^{\mu \alpha^{\prime \prime}} D_{\beta^{\prime}} \overleftarrow{X}_{\alpha \beta}^{\nu}\right] Q_{2}, \\
& t_{A C}=-\frac{g^{\alpha \beta} g_{\mu \nu}}{2} \operatorname{tr}\left[\vec{X}_{\alpha \beta}^{\mu} \overleftarrow{X}_{01}^{\nu}+\vec{X}_{01}^{\mu} \overleftarrow{X}_{\alpha \beta}^{\nu}+\vec{X}_{\alpha \beta}^{\mu} \overleftarrow{X}_{03 Z}^{\nu}+\vec{X}_{03 Z}^{\mu} \overleftarrow{X}_{\alpha \beta}^{\nu}\right. \\
& \left.-\partial_{\alpha^{\prime}} \vec{X}_{\alpha \beta}^{\mu} \breve{X}_{03 Y}{ }^{\nu \alpha^{\prime}}-\vec{X}_{03 Y}^{\mu \alpha^{\prime}} \partial_{\alpha^{\prime}} \breve{X}_{\alpha \beta}^{\nu}\right] Q_{2} \\
& -\frac{1}{4} g^{\alpha \beta} \operatorname{tr}\left[\vec{X}_{01}^{\mu} \overleftarrow{X}_{\alpha \beta}^{\mu} H_{\xi, 1}+\vec{X}_{01}^{\mu} H_{W, 1}^{\mu \nu} \overleftarrow{X}_{\alpha \beta}^{\nu}+\vec{X}_{\alpha \beta}^{\mu} \overleftarrow{X}_{01}^{\mu} H_{\xi, 1}+\vec{X}_{\alpha \beta}^{\mu} H_{W, 1}^{\mu \nu \nu} \overleftarrow{X}_{01}^{\nu}\right] Q_{0} \\
& +g_{\mu \nu}\left(\frac{1}{6} g^{\alpha \beta \alpha^{\prime} \beta^{\prime}}-\frac{1}{4} g^{\alpha \beta} g^{\alpha^{\prime} \beta^{\prime}}\right) \operatorname{tr}\left[\vec{X}_{\alpha \beta}^{\mu} D_{\alpha^{\prime}} D_{\beta^{\prime}} \overleftarrow{X}_{01}^{\nu}+\vec{X}_{01}^{\mu} D_{\alpha^{\prime}} D_{\beta^{\prime}} \overleftarrow{X}_{\alpha \beta}^{\nu}\right] Q_{0}, \\
& t_{B B}=-\frac{g_{\mu \nu} g_{\alpha \beta}}{2} \operatorname{tr}\left[\vec{X}^{\mu \alpha} \stackrel{\leftarrow}{X}\right] Q_{2}, \\
& t_{B C}=\frac{g^{\alpha \beta} g_{\alpha \alpha^{\prime}} g_{\mu \nu}}{2} \operatorname{tr}\left[\vec{X}^{\mu \alpha^{\prime}} D_{\beta} \overleftarrow{X}_{01}^{\nu}-\vec{X}_{01}^{\mu} D_{\beta} \overleftarrow{X}^{\nu \alpha^{\prime}}\right] Q_{0} \\
& t_{C C}=g_{\mu \nu} \operatorname{tr}\left[\vec{X}_{01}^{\mu} \overleftarrow{X}_{01}^{\nu}+\vec{X}_{01}^{\mu} \overleftarrow{X}_{03 Z}^{\nu}+\vec{X}_{03 Z}^{\mu} \overleftarrow{X}_{01}^{\nu}-\partial_{\alpha^{\prime}} \vec{X}_{01}^{\mu} \overleftarrow{X}_{03 Y}^{\nu \alpha^{\prime}}-\vec{X}_{03 Y}^{\mu \alpha^{\prime}} \partial_{\alpha^{\prime}} \overleftarrow{X}_{01}^{\nu}\right] Q_{0}
\end{aligned}
$$

The divergent functions $Q_{i}$ represent quartic, quadratic and logarithmic divergences, respectively, which are given as

$$
\begin{aligned}
Q_{4} & =\left(m_{W}^{2}\right)^{\frac{d}{2}} \Gamma\left(-\frac{d}{2}\right) \\
& =\frac{m_{W}^{4}}{2}\left\{\frac{2}{\epsilon}+\frac{3}{2}+\ln \left(\frac{\mu^{2}}{m_{W}^{2}}\right)\right\}, \\
Q_{2} & =\left(m_{W}^{2}\right)^{\frac{d}{2}-1} \Gamma\left(1-\frac{d}{2}\right) \\
& =-m_{W}^{2}\left\{\frac{2}{\epsilon}+1+\ln \left(\frac{\mu^{2}}{m_{W}^{2}}\right)\right\},
\end{aligned}
$$




$$
\begin{aligned}
Q_{0} & =\left(m_{W}^{2}\right)^{\frac{d}{2}-2} \Gamma\left(2-\frac{d}{2}\right) \\
& =\frac{2}{\epsilon}+\ln \left(\frac{\mu^{2}}{m_{W}^{2}}\right) .
\end{aligned}
$$

The terms $\vec{X}_{01}^{\mu} H_{W, 1}^{\mu \nu} \overleftarrow{X}_{\alpha \beta}^{\nu}$ and $\vec{X}_{\alpha \beta}^{\mu} \overleftarrow{X}_{01}^{\mu} H_{\xi, 1}$ in $t_{A C}$ given in Eq. (C.3e) are missing in the reference [9], which will modified the $\beta$ functions of $d_{i}$. However, this modification does not change the conclusion of reference [9].

\section{APPENDIX D: SCALAR LOOP INTEGRALS IN THE COORDINATE SPACE}

Here we discuss the interpretation of scalar $B_{0}$ integrals in context of the coordinate space calculations and compare it with the momentum space calculations.

In order to establish the correspondence of a scalar integral, here we consider the real scalar $\phi^{4}$ theory. The Lagrangian of this model is given as

$$
L=\frac{1}{2} \partial \phi \cdot \partial \phi+\frac{1}{2} \phi^{2}+\frac{\lambda}{4} \phi^{4} .
$$

In the BFM, we decompose $\phi$ into classical and quantum parts as $\phi=\bar{\phi}+\widehat{\phi}$. Since we only consider the one-loop corrections, the only relevant terms in the Lagrangian is the quadratic terms, which can be formulated as

$$
\begin{aligned}
L_{\text {quad }} & =\frac{1}{2} \widehat{\phi} \square \hat{\phi}, \\
\square & =-\partial^{2}+m^{2}+\sigma, \\
\sigma & =\frac{1}{2} \bar{\phi}^{2} .
\end{aligned}
$$

After performing the path integral, the one-loop effective Lagrangian can be represented as

$$
L_{\text {eff }}^{1-l o o p}=-\frac{1}{2} \operatorname{Tr} \ln \square .
$$

To evaluate $\operatorname{Tr} \ln \square$, by using the heat kernel method in coordinate space, we have

$$
\begin{aligned}
\operatorname{Tr} \ln \square= & \int_{x} \ln \left[-\partial^{2}+m^{2}+\sigma\right] \\
= & \int_{x} \ln \left[-\partial^{2}+m^{2}\right]+\int_{x} \ln \left[1+\sigma\left(-\partial^{2}+m^{2}\right)^{(-1)}\right] \\
= & \int_{x} \ln \left[-\partial^{2}+m^{2}\right]-\int_{x} \sigma\left(-\partial^{2}+m^{2}\right)^{(-1)} \\
& +\frac{1}{2} \int_{x} \sigma\left(-\partial^{2}+m^{2}\right)^{(-1)} \sigma\left(-\partial^{2}+m^{2}\right)^{(-1)}+\cdots \\
= & l_{f}\left\{\left(m^{2}\right)^{\frac{d}{2}} \Gamma\left(-\frac{d}{2}\right)-\int_{x} \sigma(x) A\left(m^{2}\right)+\frac{1}{2} \int_{x} \sigma^{2}(x) B_{0}\left(0, m^{2}, m^{2}\right)+\cdots\right\} .
\end{aligned}
$$


Now we evaluate $\operatorname{Tr} \ln \square$ in momentum space,

$$
\begin{aligned}
\operatorname{Tr} \ln \square= & \int_{x} \ln \left[-\partial^{2}+m^{2}\right]-\int_{x} \sigma\left(-\partial^{2}+m^{2}\right)^{(-1)} \\
& +\frac{1}{2} \int_{x} \sigma\left(-\partial^{2}+m^{2}\right)^{(-1)} \sigma\left(-\partial^{2}+m^{2}\right)^{(-1)}+\cdots \\
= & \int_{k} \ln \left[k^{2}+m^{2}\right]-\int_{k} \tilde{\sigma}\left(k^{2}+m^{2}\right)^{(-1)} \\
& +\frac{1}{2} \int_{k} \tilde{\sigma}\left(k_{1}, p-k_{1}\right)\left(k^{2}+m^{2}\right)^{(-1)} \tilde{\sigma}\left(k_{1}^{\prime},-p-k_{1}^{\prime}\right)\left((k+p)+m^{2}\right)^{(-1)}+\cdots \\
= & l_{f}\left\{\left(m^{2}\right)^{\frac{d}{2}} \Gamma\left(-\frac{d}{2}\right)-\int_{k_{1}} \tilde{\sigma}\left(k_{1},-k_{1}\right) A\left(m^{2}\right)\right. \\
& \left.+\frac{1}{2} \int_{k_{1}} \int_{k_{1}^{\prime}} \int_{p} \tilde{\sigma}\left(k_{1}, p-k_{1}\right) \tilde{\sigma}\left(k_{1}^{\prime},-p-k_{1}^{\prime}\right) B_{0}\left(p^{2}, m^{2}, m^{2}\right)+\cdots\right\},
\end{aligned}
$$

where $\tilde{\sigma}$ is given as

$$
\tilde{\sigma}\left(k_{1}, k_{2}\right)=\int_{k_{1}} \int_{k_{2}} \bar{\phi}\left(k_{1}\right) \bar{\phi}\left(k_{2}\right) \delta^{d}\left(k_{1}+k_{2}+p_{1}+p_{2}\right) .
$$

Here $p_{1}$ and $p_{2}$ are the momentum of the quantum fields $\widehat{\phi}$ respectively. $\bar{\phi}\left(k_{1}\right)$ is the Fourier transformation of the background field $\bar{\phi}$.

We find that if we expand the $B_{0}\left(p^{2}, m^{2}, m^{2}\right)$ given in Eq.(D.5) with reference to $p^{2}$, which appeared in the momentum space calculation, the $B\left(0, m^{2}, m^{2}\right)$ given in Eq.(D.4) is essentially the first term of this expansion, which appeared in the coordinate space calculation.

Here we also provide some results of this scalar integral $B_{0}\left(p^{2}, m_{1}^{2}, m_{2}^{2}\right)$ [22], which are used in section 6 to make one to one correspondence to the results of reference [2] in the decoupling limit. $B_{0}\left(p^{2}, m_{1}^{2}, m_{2}^{2}\right)$ can be exactly expressed as

$$
B_{0}\left(p^{2}, m_{1}^{2}, m_{2}^{2}\right)=\frac{2}{\bar{\epsilon}}+2-\ln \left(\frac{m_{1} m_{2}}{\mu^{2}}\right)+\frac{m_{1}^{2}-m_{2}^{2}}{p^{2}} \ln \left(\frac{m_{1}}{m_{2}}\right)-\frac{m_{1} m_{2}}{p^{2}}\left(\frac{1}{r}-r\right) \ln (r),
$$

where $r$ satisfies

$$
x^{2}+\frac{m_{1}^{2} m_{2}^{2}-p^{2}-i \epsilon}{m_{1} m_{2}} x+1=(x+r)\left(x+\frac{1}{r}\right) .
$$

Here we list the values of $B_{0}\left(p^{2}, m_{1}^{2}, m_{2}^{2}\right)$ in some special cases that we have used in evaluating the anomalous couplings at the one loop level:

Case 1: In the coordinate space calculation, as we have learnt that a scalar one loop $B_{0}$ integral can be defined with external momentum $p^{2}=0$ and internal masses $m_{i}, i=1,2$ as 


$$
B_{0}\left(0, m_{1}^{2}, m_{2}^{2}\right)=\frac{2}{\bar{\epsilon}}+1-\frac{r_{2} \ln r_{2}-r_{1} \ln r_{1}}{r_{2}-r_{1}}
$$

where $r_{i}=m_{i}^{2} / \mu_{E}^{2}$.

Case 2: In the coordinate space, the contribution from the Figs. 1(a),1(b) and 1(c) can be realized with $p^{2}=0$, and $m_{1}=m_{2}$ as

$$
B_{0}\left(0, m_{1}^{2}, m_{1}^{2}\right)=\frac{2}{\bar{\epsilon}}-\ln r_{1}
$$

Case 3: However, in the momentum space, working in Landau gauge the scalar integral corresponding to the Fig. $1(\mathrm{~d})$ can be realized with $m_{1}=m_{2}=0$ as

$$
B_{0}\left(p^{2}, 0,0\right)=\frac{2}{\bar{\epsilon}}-\ln \left(\frac{p^{2}}{\mu_{E}^{2}}\right)+2+i \pi .
$$

Case 4: Similarly, in the momentum space, working in On-Shell renormalization scheme would render the contribution from the Fig. 1(e) with $p^{2}=m_{1}^{2}=m_{2}^{2}=m^{2}$ as

$$
B_{0}\left(m^{2}, m^{2}, m^{2}\right)=\frac{2}{\bar{\epsilon}}-\ln \left(\frac{m^{2}}{\mu_{E}^{2}}\right)+2-\frac{\pi}{\sqrt{3}}
$$

[1] H. Georgi, Annu. Rev. Nucl. Part. Sci. 43, 209 (1993).

[2] S. Dittmaier and C. Grosse-Knetter, Phys. Rev. D 52, 7276 (1995).

[3] T. Appelquist, and C. Bernard, Phys. Rev. D 22, 200 (1980); ibid D 23, 425 (1981); J. Gasser, and H. Leutwyler, Nucl. Phys. B250, 465 (1985); ibid Ann. Phys. (N.Y.) 158, 142 (1984); T. Appelquist, Proceedings of the 21st Scottish Universities Summer School in Physics ( 1981 ).

[4] A. C. Longhitano, Phys. Rev. D 22, 1166 (1980); Nucl. Phys. B188 118 (1981); T. Appelquist, and G. H. Wu, Phys. Rev. D 48, 3235 (1993); K. Hagiwara, R.D. Peccei, and D. Zeppenfeld, Nucl. Phys. B282, 253, (1987).

[5] A. Pich, hep-ph/9806303. 
[6] M. J. Herrero, and E. R. Morales, Nucl. Phys. B418, 431 (1994); ibid B437, 319 (1995). D. Espriu and J. Matias, Phys. Lett. B341, 332 (1995).

[7] M. Bilenky and A. Santamaria, Nucl. Phys. B420, 47 (1994).

[8] S. Dittmaier and C. Grosse-Knetter, Nucl. Phys. B459, 497 (1996).

[9] Q. S. Yan and D.S. Du, Phys. Rev. D69, 085006 (2004).

[10] C. Lee, Nucl. Phys. B207, 157 (1982).

[11] P. W. Higgs, Phys. Rev. 145, 1156 (1966).

[12] R. Haag, Phys. Rev. 112, 669 (1958); S. Coleman, J. Wess, and B. Zumino, Phys. Rev. 177, 2239 (1969); C. G. Callen, S. Coleman, J. Wess, and B. Zumino, Phys. Rev. 177, 2247 (1969).

[13] K. A. Woodhouse, Nuovo Cimento A 23, 459 (1973).

[14] C. Grosse-Knetter, and R. Kögerler, Phys. Rev. D 48, 2865 (1993).

[15] S. Weinberg, The Quantum Theory of Fields (Cambridge University Press, N.Y. 2000), Vol. I, Chapter 12.

[16] K. G. Wilson and J. Kogut, Phys. Repts. 12 C, 75 (1974).

[17] J. Polchinski, Nucl. Phys. B231, 269 (1984).

[18] B.S. DeWitt, Phys. Rev. 162, 1195 (1967); ibid, 162, 1239 (1967) .

[19] C. Grosse-Knetter, Phys. Rev. D 49, 6709 (1994);

[20] H. Georgi, Nucl. Phys. B 361, 339 (1991); C. Arzt, Phys. Lett. B 342 , 189 (1995); S. Scherer and H. W. Fearing, Phys. Rev. D 52 , 6445 (1995).

[21] I.G. Avramidi, Nucl. Phys. B355, 712 (1991); Erratum-ibid. B509, 577 (1998).

[22] A. Denner, Fortschr. Phys. 41, 307 (1993). 